\title{
Feedback stabilisation of locally controllable systems $^{1}$
}

\author{
Pantelis Isaiah $^{2}$
}

$2012 / 09 / 24$

\footnotetext{
${ }^{1} \mathrm{PhD}$ thesis, Department of Mathematics and Statistics, Queen's University

${ }^{2}$ Postdoctoral Fellow, Aerospace Engineering, Technion, Israel

Email: pantelis@mast .queensu.ca

Work performed while a graduate student at Queen's University.
} 


\section{Abstract}

Controllability and stabilisability are two fundamental properties of control systems and it is intuitively appealing to conjecture that the former should imply the latter; especially so when the state of a control system is assumed to be known at every time instant. Such an implication can, indeed, be proven for certain types of controllability and stabilisability, and certain classes of control systems. In the present thesis, we consider real analytic control systems of the form $\Sigma: \dot{x}=f(x, u)$, with $x$ in a real analytic manifold and $u$ in a separable metric space, and we show that, under mild technical assumptions, smalltime local controllability from an equilibrium $p$ of $\Sigma$ implies the existence of a piecewise analytic feedback $\mathscr{F}$ that asymptotically stabilises $\Sigma$ at $p$. As a corollary to this result, we show that nonlinear control systems with controllable unstable dynamics and stable uncontrollable dynamics are feedback stabilisable, extending, thus, a classical result of linear control theory.

Next, we modify the proof of the existence of $\mathscr{F}$ to show stabilisability of small-time locally controllable systems in finite time, at the expense of obtaining a closed-loop system that may not be Lyapunov stable. Having established stabilisability in finite time, we proceed to prove a converse-Lyapunov theorem. If $\mathscr{F}$ is a piecewise analytic feedback that stabilises a small-time locally controllable system $\Sigma: \dot{x}=f(x, u)$ in finite time, then the Lyapunov function we construct has the interesting property of being differentiable along every trajectory of the closed-loop system obtained by "applying" $\mathscr{F}$ to $\Sigma$.

We conclude this thesis with a number of open problems related to the stabilisability of nonlinear control systems, along with a number of examples from the literature that hint at potentially fruitful lines of future research in the area. 
P. IsAiAh

To the chimæras of the past 


\section{Contents}

1 Introduction 1

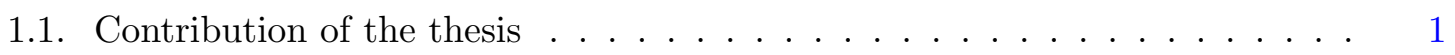

1.2. A few motivating examples .................. 1

2 Literature review 5

2.1. General theorems on stabilisation . . . . . . . . . . . . 5

2.1.1 One-dimensional control systems. . . . . . . . . . . 5

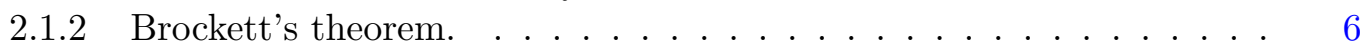

2.1.3 Coron's theorem. . . . . . . . . . . ..... 8

2.1.4 Artstein's theorem. . . . . . . . . . . . . . . . 9

2.2. Stabilisation under controllability assumptions . . . . . . . . . . . 11

2.2.1 Locally controllable systems. . . . . . . . . . . . . . . . . . . . . . 12

2.2.2 Globally controllable systems. . . . . . . . . . . . . . . . 16

2.2.3 Asymptotically controllable systems. . . . . . . . . . . . . . 17

3 Feedback stabilisation of locally controllable systems 22

3.1. Introduction . . . . . . . . . . . . . . . . . . 22

3.2. Real analytic geometry . . . . . . . . . . . . . . . . . . . . . 22

3.2.1 Semi-analytic and subanalytic sets. . . . . . . . . . . . 23

3.2 .2 Stratifications. . . . . . . . . . . . . . . . . . . 23

3.3. Control theory . . . . . . . . . . . . . . . . . . . 24

3.3 .1 Piecewise analytic feedback. . . . . . . . . . . . . . 24

3.3 .2 Normal reachability. . . . . . . . . . . . . . . . . 25

3.4. Proof of the main result . . . . . . . . . . . . . . . 25

3.5. A corollary . . . . . . . . . . . . . . . . . . 29

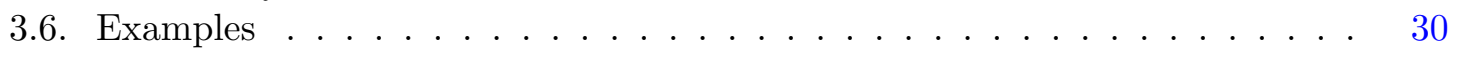

4 A converse Lyapunov-type theorem for locally controllable systems 31

4.1. Introduction . . . . . . . . . . . . . . . . . . 31

4.1.1 Lyapunov stability and converse Lyapunov theorems. . . . . . . . . . 31

4.1 .2 Zubov's method. . . . . . . . . . . . . . . . . . 32

4.1 .3 Control theory. . . . . . . . . . . . . . . . 33

4.2. Stabilisation of locally controllable systems in finite time . . . . . . . . . 34

4.3. Proof of the main result . . . . . . . . . . . . . . . . 36

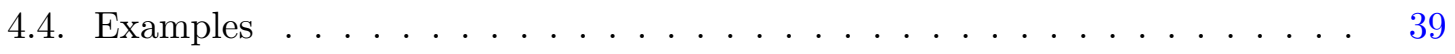

5 Future work $\quad 43$

5.1. Regularity of asymptotically stabilising feedback controls . . . . . . . . . 43

5.2 . The temporal aspect of stabilisation . . . . . . . . . . . 45

$\begin{array}{ll}\text { Index } & 51\end{array}$ 


\section{Chapter 1}

\section{Introduction}

\subsection{Contribution of the thesis}

Controllability and feedback stabilisation are two fundamental problems in control theory and this thesis establishes a connection between the two. Although it is natural to strive for utmost generality, in order to reach any meaningful conclusion it is necessary to work within the boundaries of a precisely defined framework which, in our case, is provided by the class of real analytic control systems that are small-time locally controllable from an equilibrium point $p$ and that can be represented in coordinates by an expression of the form $\dot{x}=f(x, u)$. Within this framework, a feedback is understood to be a vector field that can be realised by assigning values to the control parameters. A stabilising feedback is, then, a feedback whose trajectories converge to $p$ asymptotically or in finite time. After these notions are made precise we show that, under an additional assumption that guarantees the compactness of the reachable sets, small-time local controllability implies the existence of an asymptotically stabilising piecewise analytic feedback. As a corollary, we obtain a nonlinear analogue of the stabilisability of linear control systems with controllable unstable eigenvalues. Next, by modifying the proof of asymptotic stabilisability, we show that smalltime locally controllable systems can also be stabilised in finite time and, building on this fact, we prove a converse Lyapunov theorem. That is, we show that, in a neighbourhood of the equilibrium $p$, there exists a function which is positive definite, continuous at $p$, and its derivative along the trajectories of the closed-loop system is negative.

\subsection{A few motivating examples}

One of the challenges in understanding and classifying nonlinear control systems according to their stabilisability properties is that there exist obstructions to stabilisation that are genuinely nonlinear, in the sense that they have no analogues in the linear theory. The examples in this section are meant to demonstrate this observation in a concrete manner and, also, to show that the relationship between nonlinear controllability and stabilisation is, at best, indirect. Moreover, the examples that conclude the section indicate that some basic questions on stabilisation remain elusive, despite their deceivingly simple formulation. Unlike the rest of the thesis, in this section we freely use terms that have not yet been defined and we let the reader assign an intuitive meaning to them based on the context.

We begin by recalling an important aspect of the stabilisation problem for linear control 
systems that has been well understood for some time now. Let $A \in L\left(\mathbb{R}^{n} ; \mathbb{R}^{n}\right), B \in$ $L\left(\mathbb{R}^{m} ; \mathbb{R}^{n}\right)$, and consider the control system

$$
\dot{x}=A x+B u,
$$

with the admissible controls being the locally integrable maps $[0, \infty) \ni t \mapsto u(t) \in \mathbb{R}^{m}$. Aslo, let $\lambda$ denote a complex number, $\mathbb{C}_{-}$the open left-half of the complex plane, $\operatorname{rank} M$ the rank of a matrix $M$, and $\sigma(M)$ the spectrum of a matrix $M$. Then, according to a theorem proven by Hautus, the following statements are equivalent [46]:

(controllability of unstable eigenvalues)

(stabilisability)

(asymptotic controllability)

$$
\begin{gathered}
\Re(\lambda) \geq 0 \Rightarrow \operatorname{rank}[A-\lambda I \mid B]=n \Leftrightarrow \\
\exists K \in L\left(\mathbb{R}^{n} ; \mathbb{R}^{m}\right) \mid \sigma(A+B K) \subset \mathbb{C}_{-} \Leftrightarrow
\end{gathered}
$$

The system (L) is asymptotically controllable to $0 \in \mathbb{R}^{n}$.

For emphasis, let us state in words two of the conclusions that can be drawn from the above equivalences. First, the only obstruction to static, state dependent, asymptotic stabilisation of finite dimensional linear control systems of the form (L) is the existence of uncontrollable eigenvalues with non-negative real part. Second, if the states of a linear control system can be driven to the origin by open loop controls, then, not only does there exist a stabilising feedback, but one that is linear. This implies that if a linear system can be stabilised by a feedback of any regularity (always assuming the local integrability of the controls), then the system can also be stabilised with a linear, and hence $C^{\infty}$, feedback. It will become clear below that a similar conclusion is far from being true for nonlinear control systems. However, its validity in the linear case has motivated significant results on nonlinear stabilisation, some of which are described in Section 2.2.3. It is worth mentioning that linear systems also form a tractable class of control systems when other types of stabilisation are considered. For example, it can be shown that controllable linear systems can be globally stabilised in finite time using continuous feedback laws [38].

To contrast Hautus's theorem, cited above, with the case of nonlinear control systems, consider the control-affine system $\Sigma: \dot{\mathbf{x}}=X(\mathbf{x})+v Y(\mathbf{x}), \mathbf{x}=(x, y) \in \mathbb{R}^{2}, X, Y: \mathbb{R}^{2} \rightarrow \mathbb{R}^{2}$, $v \in \mathbb{R}$, where

$$
X(\mathbf{x})=\left[\begin{array}{cc}
4 & 0 \\
0 & 1
\end{array}\right] \mathbf{x} \quad \text { and } \quad Y(\mathbf{x})=\left[\begin{array}{c}
y^{2} \\
1
\end{array}\right]
$$

It can be shown by directly solving the equations of $\Sigma$ that any two points in $\mathbb{R}^{2}$ can be connected with a trajectory of $\Sigma$. In other words, $\Sigma$ is globally controllable. However, there does not exist $v \in C^{1}\left(\mathbb{R}^{2}\right)$ that renders $\Sigma$ asymptotically stable at the origin. This can be seen by noting that such a $v$ has to satisfy $v(0,0)=0$ (for otherwise the origin would not be an equilibrium) and that the linearisation has an eigenvalue with positive real part, regardless of the form of $v$ [52]. Therefore, $\Sigma$ is globally controllable, affine in the control, and has linear drift, however it cannot be asymptotically stabilised at the origin using $C^{1}$ feedback. ${ }^{1}$

\footnotetext{
${ }^{1}$ The Jurdjevic-Quinn theory of stabilization, initiated in [52], has been generalised to systems with nonlinear drift. However, our goal here is precisely to show how even "simple" nonlinear systems deviate from the linear theory.
} 
Next, we look at the driftless control system $\Sigma: \dot{\mathbf{x}}=u X(\mathbf{x})+v Y(\mathbf{x}), \mathbf{x}=(x, y, z) \in \mathbb{R}^{3}$, $X, Y: \mathbb{R}^{3} \rightarrow \mathbb{R}^{3}, u, v \in \mathbb{R}$, defined by

$$
X(\mathbf{x})=\left[\begin{array}{c}
1 \\
0 \\
-y
\end{array}\right] \quad \text { and } \quad Y(\mathbf{x})=\left[\begin{array}{l}
0 \\
1 \\
x
\end{array}\right]
$$

and known in the literature as "Brockett's integrator". The control system $\Sigma$ is locally controllable ${ }^{2}$ from $0 \in \mathbb{R}^{3}$, as well as globally and asymptotically controllable. Nevertheless, Brockett showed in $[15$, p. 181] that $\Sigma$ violates a topological condition that is necessary for the existence of a $(u, v) \in C^{1}\left(\mathbb{R}^{3} ; \mathbb{R}^{2}\right)$ that makes $0 \in \mathbb{R}^{3}$ an asymptotically stable equilibrium. Zabczyck [87] and Ryan [72] later showed that the same topological condition persists also when larger classes of feedback controls are allowed. We revisit Brockett's result and its extensions in Section 2.1.2.

The previous two examples show that some common notions of controllability for nonlinear systems do not imply stabilisability. A natural question to ask is whether the opposite implication is true, that is, whether stabilisability implies controllability. In general, this is not true even for one-dimensional systems. For example, consider the control system $\Sigma: \dot{x}=(1-u) x, x, u \in \mathbb{R}$. Any constant control $u=1+\varepsilon, \varepsilon>0$, yields a globally asymptotically stable closed-loop, however, for reasonable classes of admissible controls, e.g., piecewise continuous $u: \mathbb{R} \rightarrow \mathbb{R}, \Sigma$ is neither locally controllable from $0 \in \mathbb{R}$ nor globally controllable.

The next four examples of control systems that conclude this introductory section are meant to illustrate the fact that deciding the existence of a stabilising feedback is only one facet of the problem of static, state feedback stabilisation for finite dimensional, nonlinear control systems; once it is known that a control system is stabilisable, there are natural questions to ask concerning the regularity and robustness of a stabilising feedback. In this regard, consider the problem of asymptotically stabilising at the origin $0 \in \mathbb{R}^{2}$ the control systems

$$
\text { (A) }\left\{\begin{array}{l}
\dot{x}=u, \\
\dot{y}=y-x^{3},
\end{array}\right.
$$

$$
\left\{\begin{array}{l}
\dot{x}=u \\
\dot{y}=y^{2}-x^{4}
\end{array}\right.
$$

where $x, y, u \in \mathbb{R}$. The following statements are true:

- There exists a Hölder-continuous map $u: \mathbb{R}^{2} \rightarrow \mathbb{R}$ that stabilises (A).

- There does not exist $u \in C^{1}\left(\mathbb{R}^{2}\right)$ that stabilises $(\mathrm{A})$.

- There exists $u \in C^{1}\left(\mathbb{R}^{2}\right)$ that stabilises (B).

- There does not exist $u \in C^{3}\left(\mathbb{R}^{2}\right)$ that stabilises (B).

The proof of the first statement can be found in [53] and will be reviewed in Section 2.2.1. The second statement follows from Lyapunov's first method, that is, from a linearisation argument, whereas the proof of the last two statements is contained in [30]. Systems (A)

\footnotetext{
${ }^{2}$ Locally controllable means that, by following the trajectories of the control system for all admissible controls and for arbitrarily small time, it is possible to reach a neighbourhood of $0 \in \mathbb{R}^{3}$.
} 
and (B) are formally similar - they are both control-affine, with polynomial drift, and of the same dimension - however a variety of techniques has to be employed to prove the stabilisability properties listed above. An interesting problem is to devise a method that can distinguish between (A) and (B), based on their stabilisability properties. Finallly, consider the problem of asymptotically stabilising at the origin $0 \in \mathbb{R}^{3}$ the control systems

$$
\text { (C) }\left\{\begin{array} { l } 
{ \dot { x } = u , } \\
{ \dot { y } = v , } \\
{ \dot { z } = x ^ { 2 } - y ^ { 2 } , }
\end{array} \quad \text { (D) } \left\{\begin{array}{l}
\dot{x}=u, \\
\dot{y}=x^{2}(y-x), \\
\dot{z}=x^{2}(z-x),
\end{array}\right.\right.
$$

assuming that the set of control values is convex and contains $0 \in \mathbb{R}^{3}$ in its interior. The following facts can be proven:

- there exist polynomial maps $u$ and $v$ that stabilise $(\mathrm{C})$;

- there exists a piecewise analytic feedback that stabilises (D);

- there does not exist $u \in C^{0}\left(\mathbb{R}^{3}\right)$ that stabilises (D).

The proofs follow from Centre Manifold theory, Theorem 3.3, and the results in [26], respectively. It will be seen later in the text that systems (C) and (D), besides both being control-affine, with polynomial drift, and of the same dimension, also share certain controllability properties. As in the case of systems (A) and (B), it is not clear how to systematically identify the structure that dictates the different stabilisability properties.

The problem of characterising the regularity of stabilising feedbacks for a given control system is not resolved in this thesis; in Chapter 5.1, using results from the literature as supporting evidence, we hint at directions that could lead to progress on the solution of this problem. In any case, a conclusion that can be safely drawn, and which is one of the goals of the present discussion, is that the problem of feedback stabilisation for nonlinear control systems, even when confined to static state feedback for finite dimensional deterministic systems of the form $\dot{x}=f(x, u)$, can be considered as far from being settled. 


\section{Chapter 2}

\section{Literature review}

\subsection{General theorems on stabilisation}

Unless one is confined to specific classes of control systems and types of stabilisation, the literature on stabilisation of control systems is too vast - see, e.g., $[6,7,51]$ and the references therein - to review any representative body of results. Therefore, we adopt the following criteria as to what results to include here and what not to include. First, we consider results because of their generality in the sense that they may require certain assumptions on the regularity of the equations that define a control system, but otherwise they impose no restrictions on the equations other than being of the form $\dot{x}=f(x, u)$. Results of this type are reviewed in Section 2.1. Second, we omit results that impose conditions on a control system that make the problem of local asymptotic feedback stabilisation trivial. Most notably, results that require the linearisation of a nonlinear control system to be controllable, e.g., the method of feedback linearisation [67, Ch. 6] and techniques based on bifurcation theory $[1,2]$. Third, we restrict our attention to results that are similar in philosophy to ours. To wit, results that establish connections between controllability and stabilisation and are as relevant as possible to the problem of static, state feedback stabilisation of nonlinear, finite dimensional control systems of the form $\dot{x}=f(x, u)$. This rule is not strictly followed since there are interesting results, e.g., Theorem 2.17 and Theorem 2.24, on the stabilisation of controllable systems that involve time-dependent or sampled-data feedbacks; however, we do categorise the results in Sections 2.2.1 and 2.2.3 according to the type of controllability assumed for a control system. The effect of this third criterion is that, for example, we exclude the parts of the literature that address the problems of output feedback stabilisation, of stabilisation of infinite dimensional systems, or the stabilisation of systems that cannot be expressed in the form $\dot{x}=f(x, u)$.

2.1.1. One-dimensional control systems. Obstructions to stabilisation exist even for onedimensional control systems. If regularity, e.g., continuity, of feedback is required, then one can construct examples of one-dimensional control systems that (1) can be semi-globally, but not globally, stabilised, (2) that can only be stabilised with controls that undergo an infinite number of switchings, or (3) that depend on the control in a nonlinear fashion that imposes algebraic obstructions on continuous stabilisability [75]. More generally, the

pathologies that can be constructed are only limited by one's ability to imagine functions with complicated level sets. On the other hand, due to the simplicity of the state space, it 
is possible to characterise the (one-dimensional) control systems that are stabilisable.

The basic idea introduced in [76] in connection with one-dimensional control systems and their stabilisability properties is to express the latter in terms of the graph of a feedback control. To demonstrate this idea we make a simplifying assumption on the regularity of feedbacks and refer the reader to [76] for a more general definition of a stabilising feedback and for other results on one-dimensional control systems (see also Section 2.2.3).

Consider a control system of the form $\Sigma: \dot{x}=f(x, u), x, u \in \mathbb{R}$, and suppose that $u: \mathbb{R} \rightarrow \mathbb{R}$, with $u(0)=0$, is a map such that $x \mapsto f(x, u(x))$ is locally Lipschitz and $x=0$ is an asymptotically stable equilibrium of the dynamical system $\dot{x}=f(x, u(x))$. Then, if we define

$$
\mathcal{O} \triangleq\left\{(x, v) \in \mathbb{R}^{2} \mid x f(x, v)<0\right\},
$$

asymptotic stability of $x=0$ implies that the graph of $u$ must lie in $\mathcal{O}$. Conversely, if there exists a $u$ as above and the condition on the graph is satisfied, then $\Sigma$ is stabilisable. Using this observation, we can construct a simple one-dimensional control system that does not admit a stabilising feedback $u$ with the above properties.

2.1 Example: The control system

$$
\dot{x}=x\left[2(u+1)^{2}+(x-1)\right]\left[2(u-1)^{2}-(x+1)\right]
$$

cannot be asymptotically stabilised at $x=0$ using a feedback $u: \mathbb{R} \rightarrow \mathbb{R}$ that satisfies $u(0)=0$ and is locally Lipschitz in a neighbourhood of $x=0$. Since $\operatorname{pr}_{1} \mathcal{O}=\mathbb{R}$, the system (2.1.1) is asymptotically controllable to $x=0$. However, it cannot be globally stabilised with a feedback $u \in C^{0}(\mathbb{R})$. On the other hand, it can be easily verified that if $u=-1$, then (2.1.1) becomes $\dot{x}=-x^{3}+8 x^{2}-7 x$, which is a locally asymptotically stable vector field.

2.1.2. Brockett's theorem. Brockett's theorem is one of the earliest general results on feedback stabilisation of nonlinear control systems. It is a necessary condition that a system of the form $\dot{x}=f(x, u)$ must satisfy in order to be stabilisable using smooth feedback. More precisely, suppose $f: \mathbb{R}^{n} \times \mathbb{R}^{m} \rightarrow \mathbb{R}^{n}$ is map that is continuously differentiable in a neighbourhood of a point $\left(x_{e q}, 0\right)$ and that satisfies $f\left(x_{e q}, 0\right)=0$. The following is proven in $[15$, p. 181].

2.2 Theorem: Necessary conditions for the existence of a function $u \in C^{1}\left(\mathbb{R}^{n} ; \mathbb{R}^{m}\right)$ such that $x_{e q}$ is an asymptotically stable equilibrium of $\dot{x}=f(x, u(x))$ are that:

(i) there exists a neighbourhood $N$ of $x_{e q}$ such that for each $\xi \in N$ there exists a control $u_{\xi}:[0, \infty) \rightarrow \mathbb{R}^{m}$ such that the solution $x_{u_{\xi}}$ of the initial value problem $\dot{x}(t)=$ $f\left(x(t), u_{\xi}(t)\right), x(0)=\xi$, satisfies $\lim _{t \rightarrow+\infty} x_{u_{\xi}}(t)=x_{e q}$;

(ii) the linear system $\dot{x}=A x+B u$, where $A=\left.\frac{\partial f}{\partial x}\right|_{\left(x_{e q}, 0\right)}$ and $B=\left.\frac{\partial f}{\partial u}\right|_{\left(x_{e q}, 0\right)}-i . e$. , the linearisation of $\dot{x}=f(x, u)$ - has no uncontrollable eigenvalues whose real part is positive;

(iii) the image of the map $\mathbb{R}^{n} \times \mathbb{R}^{m} \ni(x, u) \mapsto f(x, u) \in \mathbb{R}^{n}$ contains an open neighbourhood of $0 \in \mathbb{R}^{n}$. 
The necessity of the first part of Theorem 2.2 is obvious since every closed-loop control can also be viewed as an open loop control. The necessity of the second part is a direct consequence of Lyapunov's indirect method, however it is worth noting that if condition (ii) does not hold, only the existence of $C^{1}$ feedbacks that vanish at the equilibrium can be precluded [6, p. 28].

2.3 Example: Consider the two-dimensional, single-input, control-affine system $\Sigma: \dot{\mathbf{x}}=$ $X(\mathbf{x})+u Y(\mathbf{x}), \mathbf{x}=(x, y) \in \mathbb{R}^{2}, u \in \mathbb{R}$, with

$$
X(\mathbf{x})=\left[\begin{array}{l}
0 \\
y
\end{array}\right] \text { and } Y(\mathbf{x})=\left[\begin{array}{c}
2 x-y \\
x
\end{array}\right] .
$$

Using the notation of Theorem 2.2, we have

$$
A+B K=A=\left[\begin{array}{ll}
0 & 0 \\
0 & 1
\end{array}\right]
$$

for any $K \in \mathbb{R}^{1 \times 2}$, and, therefore, the linearisation of $\Sigma$ at $(\mathbf{x}, u)=(0,0)$ has a real positive uncontrollable eigenvalue. Nevertheless, any constant $u<-2$ yields an asymptotically stable linear vector field $X(\mathbf{x})+u Y(\mathbf{x})$, as can be seen by examining the eigenvalues of the matrix

$$
\left[\begin{array}{cc}
2 u & -u \\
u & 1
\end{array}\right]
$$

2.4 Remark: The system $\Sigma$ of Example 2.3 belongs to a class of systems called "bilinear" and there exist several sufficient conditions in the literature that imply the stabilisability of bilinear systems using constant feedback [6, Ch. II].

Kawski has shown that violation of the second condition of Theorem 2.2 does not imply the non-existence of continuous stabilising feedbacks either [53].

2.5 Example: Suppose that in Example 2.3 we set

$$
X(\mathbf{x})=\left[\begin{array}{c}
0 \\
y-x^{3}
\end{array}\right] \text { and } Y(\mathbf{x})=\left[\begin{array}{l}
1 \\
0
\end{array}\right] .
$$

Then, because any feedback $u: \mathbb{R}^{2} \rightarrow \mathbb{R}$ that stabilises $\Sigma$ at $(x, y)=(0,0)$ has to satisfy $u(0,0)=0$ - for otherwise $(0,0)$ would not be an equilibrium - and because $\lambda=1$ is an uncontrollable eigenvalue of the linearisation of $\Sigma$, condition (ii) of Theorem 2.2 implies that there does not exist an asymptotically stabilising $u \in C^{1}\left(\mathbb{R}^{2}\right)$. However, it can be shown that $\Sigma$ admits a Hölder-continuous stabilising feedback [53]. In Section 2.2 we outline the idea behind the proof of this last result.

The third condition of Theorem 2.2 is of a topological nature and is far from completely characterising stabilisable systems as can be seen by considering the control system

$$
\begin{aligned}
& \dot{x}=x, \\
& \dot{y}=u,
\end{aligned}
$$

which satisfies the condition but is obviously not stabilisable by any kind of feedback $u$ : $\mathbb{R}^{2} \rightarrow \mathbb{R}$. However, system (2.1.3) is a trivial counterexample in the sense that it violates both conditions (i) and (ii) of the theorem. What is perhaps more interesting is that there exist systems that satisfy all three conditions of Theorem 2.2 and they cannot be stabilised using continuous feedback. 
2.6 Example: ${ }^{1}$ The driftless control system $\dot{\mathbf{x}}=u Y(\mathbf{x})$ with

$$
Y(\mathbf{x})=\left[\begin{array}{c}
x^{2}-y^{2} \\
2 x y
\end{array}\right]
$$

satisfies the three conditions in Theorem 2.2, however it cannot be stabilised using continuous feedback $[5,75]$.

Let us conclude this section by noting that Brockett's condition, i.e., part (iii) of Theorem 2.2 , was subsequently shown to be also necessary for the existence of a stabilising $u \in C^{0}\left(\mathbb{R}^{2} ; \mathbb{R}\right)[87]$. Moreover, it was proven in [72] that if a control system $\dot{x}=f(x, u)$, $x \in \mathbb{R}^{n}, u \in \mathbb{R}^{m}$, satisfies the condition

$$
U \subset \mathbb{R}^{m} \text { convex } \Rightarrow f(x, U) \text { convex, }
$$

for all $x \in \mathbb{R}^{n}$, Brockett's condition persists even if a feedback is understood as an upper semicontinuous set-valued map with non-empty, compact, convex values, in which case the trajectories of the control system are defined in the sense of Filippov.

2.1.3. Coron's theorem. Next, we describe another necessary topological condition for continuous feedback stabilisation of nonlinear control systems. Let $\Omega \subset \mathbb{R}^{n} \times \mathbb{R}^{m}$ be open and $f: \Omega \rightarrow \mathbb{R}^{n}$ a continuous map such that $f(0,0)=0$. A control system $\dot{x}=f(x, u)$ is said to be locally asymptotically stabilisable (LAS) if there exists a map $u \in C^{0}\left(V ; \mathbb{R}^{m}\right)$ defined on a neighbourhood $V$ of $0 \in \mathbb{R}^{n}$ such that

(i) $u(0)=0$,

(ii) there exists a neighbourhood $W \subseteq V$ of $0 \in \mathbb{R}^{n}$ such that the initial value problem $\dot{x}=f(x, u(x)), x(0) \in W$, has unique solutions, and

(iii) the origin $0 \in \mathbb{R}^{n}$ is an asymptotically stable equilibrium of the differential equation $\dot{x}=f(x, u(x))$.

If $V$ is a neighbourhood of $0 \in \mathbb{R}^{n} \times \mathbb{R}^{p}, p \in \mathbb{Z}_{>0}$, and $u \in C^{0}\left(V ; \mathbb{R}^{m}\right)$ is such that the control system (with the new control $v$ )

$$
\begin{aligned}
& \dot{x}=f(x, u(x, y)), \\
& \dot{y}=v,
\end{aligned}
$$

is LAS, then the control system $\dot{x}=f(x, u)$ is said to be dynamically locally asymptotically stabilisable (DLAS). Suppose now that $X$ is a topological space and $f: X \rightarrow \mathbb{R}^{n}$ a continuous map. Denote by $H_{k}(X), k \in \mathbb{Z}_{\geq 0}$, the $k$-th singular homology group of $X$, by $f_{*}$ the homomorphism induced on homology by $f$, and define the set

$$
\Sigma_{\varepsilon}=\{(x, u) \in \Omega \mid(|x|<\varepsilon) \wedge(|u|<\varepsilon) \wedge(f(x, u) \neq 0)\} .
$$

Coron has shown in [26] the following.

\footnotetext{
${ }^{1}$ This example has become known in the literature as "Artstein's circles".
} 
2.7 Theorem: If the system $\dot{x}=f(x, u(x))$ is LAS or DLAS, then $f_{*}\left(H_{n-1}\left(\Sigma_{\varepsilon}\right)\right)=$ $H_{n-1}\left(\mathbb{R}^{n} \backslash 0\right), \forall \varepsilon \in(0, \infty]$.

Theorem 2.7 provides a strictly stronger condition than that of Theorem 2.2.

2.8 Example: The control-affine system $\dot{\mathbf{x}}=X(\mathbf{x})+u Y(\mathbf{x}), \mathbf{x}=(x, y, z) \in \mathbb{R}^{3}, u \in \mathbb{R}$, with

$$
X(\mathbf{x})=\left[\begin{array}{c}
0 \\
z^{3}-3(y-x)^{2} z \\
(y-x)^{3}-3(y-x) z^{2}
\end{array}\right] \quad \text { and } \quad Y(\mathbf{x})=\left[\begin{array}{l}
1 \\
0 \\
0
\end{array}\right]
$$

satisfies the conditions of Theorem 2.2 [26, Prop.6], but not the condition of Theorem 2.7.

Similarly to Theorem 2.2, the condition of Theorem 2.7 is not sufficient for continuous feedback stabilisability.

2.9 Example: The control-affine system $\dot{\mathbf{x}}=X(\mathbf{x})+u Y(\mathbf{x}), \mathbf{x}=(x, y, z) \in \mathbb{R}^{3}, u \in \mathbb{R}$, where

$$
X(\mathbf{x})=\left[\begin{array}{c}
0 \\
x^{2}(y-x) \\
x^{2}(z-y)
\end{array}\right] \text { and } Y(\mathbf{x})=\left[\begin{array}{l}
1 \\
0 \\
0
\end{array}\right]
$$

satisfies the conditions of Theorems 2.2 and 2.7 and it is not LAS [26].

2.1.4. Artstein's theorem. Having seen two necessary conditions for state feedback stabilisability of nonlinear control systems, it is natural to ask when a stabilising feedback does exist or, in other words, if there exist sufficient conditions for stabilisability. One of the earliest and most general such results is a theorem due to Artstein [5]. Artstein's theorem establishes an equivalence between the existence of a continuous stabilising feedback and the existence of a smooth control-Lyapunov function. ${ }^{2}$ If the control system is of the form $\dot{x}=f(x, u)$, then the continuous feedback is a continuous map from the state space of the control system into a space of relaxed controls and if the control system is affine in the control, then the feedback is an ordinary continuous map from the state space of the control system into the metric space of control values.

Let us now sketch the ideas behind the main result in [5]. Since a closed-loop system is, in fact, a dynamical system, the existence of a smooth control-Lyapunov function for continuously stabilisable systems is a consequence of a general converse Lyapunov theorem due to Kurzweil [57]. Conversely, if such a control-Lyapunov function exists, then the existence of a continuous stabilising feedback is shown by point-wise minimisation over the admissible controls of the derivative of the control-Lyapunov function. This is exactly how relaxed controls come into play, since they form a convex space that admits a natural compact topology, two useful properties for minimisation. Artstein's theorem has several interesting aspects and corollaries for which the reader is referred to the original paper [5].

Before we proceed with the precise statement of Artstein's theorem, it is interesting to observe that, although the theorem seems to recast the problem of existence of a continuous stabilising feedback as the equally hard problem of existence of a control-Lyapunov function, it has non-trivial implications related to the regularity of stabilising feedbacks a control system admits. This aspect of Artstein's theorem will be explored further in Section 5.1.

\footnotetext{
${ }^{2} \mathrm{~A}$ control-Lyapunov function is a function that has the properties listed in Theorem 2.10.
} 
Let $U$ denote a metric space and $U_{R}$ the set of probability measures on $U$, equipped with the topology induced by the following notion of convergence. A sequence $\left(v_{k}\right)$ is said to converge to $v \in U_{R}$ if and only if

$$
\int h \mathrm{~d} v_{k} \rightarrow \int h \mathrm{~d} v
$$

for any continuous and bounded $h: U \rightarrow \mathbb{R}$. A relaxed control on $U$ is an element of $U_{R}$. 3 Consider now a control system $\Sigma: \dot{x}=f(x, u)$, where $f: \mathbb{R}^{n} \times U \rightarrow \mathbb{R}^{n}$ is a continuous map and $U$ as above. Since a relaxed control is, by definition, a probability measure, a meaning has to be assigned to the right-hand side of $\Sigma$ when a relaxed control is "applied" to $\Sigma$. To this end, if $v$ is a relaxed control, $f(x, v)$ is defined to be

$$
f(x, v) \triangleq \int_{U} f(x, u) \mathrm{d} v .
$$

Depending on the control system to be modelled, different assumptions can be made that guarantee that the right-hand side of (2.1.5) is finite [5], [37, Ch.2]. For example, one of the simplest cases is to assume that the metric space $U$ is compact. ${ }^{4}$ A relaxed feedback control can now be defined to be a map $v: N \subseteq \mathbb{R}^{n} \rightarrow U_{R}$ defined on a neighbourhood $N$ of the origin $0 \in \mathbb{R}^{n}$ and such that $x \mapsto v(x)$ and $x \mapsto f(x, v(x))$ are continuous on $N \backslash\{0\}$. If $x=0$ is an asymptotically stable equilibrium of $\Sigma: \dot{x}=f(x, v(x))$, then $v$ is a stabilising relaxed feedback control for $\Sigma$. The following result is proven in [5].

2.10 Theorem: There exists a stabilising relaxed feedback control $v: N \subseteq \mathbb{R}^{n} \rightarrow U_{R}$ for $\dot{x}=f(x, u)$ if and only if there exists a function $V \in C^{1}(N)$ such that

(i) $V(0)=0$,

(ii) $x \neq 0 \Rightarrow V(x)>0$, and

(iii) $x \neq 0 \Rightarrow \inf _{u \in U}\{\operatorname{grad} V(x) \cdot f(x, u)\}<0$.

Moreover, the function $V$ can be chosen to be in $C^{\infty}(N \backslash\{0\})$.

A function which is $C^{\infty}$ in a punctured neighbourhood of the origin will be called almost smooth. The following example is representative of how a relaxed control can fail to be continuous at the origin.

\footnotetext{
${ }^{3}$ This is the definition of a constant relaxed control; a relaxed control is, in general, a family of probability measures [37, Ch. 2]. However, the definition as it is suffices in order to state the results in [5]. In Section 2.2.3 it will be necessary to introduce a more general definition of a relaxed control. The references [37] and [85] cover the theory of relaxed controls thoroughly, with [37] being particularly clear and concise. The approach to relaxed controls taken in [86] (called "chattering controls" therein) is close to the historical development of the subject, that is, as a natural extension of the classical theory of the calculus of variations.

${ }^{4}$ It is interesting to note that, to obtain the results of the theory of relaxed controls, it suffices to consider only Dirac measures $[9,60]$. This fact hinges upon two observations: first, with respect to a suitably defined weak topology [37, p. 29], analogous to the vague topology [31, p. ], "time-varying" Dirac measures are dense in the space of relaxed controls and can be approximated arbitrarily well by piecewise constant ordinary controls; second, by a theorem due to Carathéodory [36, p. 67], any point in the convex hull of a set $A \subset \mathbb{R}^{d}$ is the convex combination of at most $d+1$ points of $A$. Therefore, any trajectory of the control system $\dot{x} \in \overline{\mathrm{co}} f(x, U)$ ( $\overline{\mathrm{co}}$ denotes the closure of the convex hull) can be approximated by a trajectory of the control system $\dot{x}=f(x, u)$ that corresponds to some piecewise constant control. If $v$ is a convex combination of Dirac measures, convergence of the integral in (2.1.5) presents no difficulties.
} 
2.11 Example: Consider the control system

$$
\Sigma: \dot{x}=(u+1)[x g(x)+2 x h(x)]+(u-1)[2 x g(x)+x h(x)],
$$

where $x \in \mathbb{R}, u \in U=\{-1,1\}$, and the functions $g, h: \mathbb{R} \rightarrow \mathbb{R}$ are defined by

$$
g(x)=\left\{\begin{array}{ll}
x, & x \leq 0, \\
0, & x>0,
\end{array} \quad \text { and } \quad h(x)= \begin{cases}0, & x \leq 0 \\
x, & x>0\end{cases}\right.
$$

There does not exist a stabilising relaxed feedback control for $\Sigma$ that is continuous at $0 \in \mathbb{R}$ since, for $x<0$, the measure assigned to $u=1$ has to be greater than $2 / 3$, whereas, for $x>0$, the measure assigned to $u=1$ has to be less than $1 / 3$.

Example 2.11 captures the general idea behind non-removable discontinuities (for stabilising feedbacks) at the origin in the sense that, if $U$ is separable and complete and $\rho$ is a convex ${ }^{5}$ semi-metric on $U_{R}$, then the relaxed control of Theorem 2.10 is $\rho$-continuous at $0 \in \mathbb{R}^{n}$ if the following property holds. ${ }^{6}$ There exists a $v_{0} \in U_{R}$ such that $f\left(0, v_{0}\right)=0$ and, for every $\varepsilon>0$, there exists a $\delta>0$ such that, if $|x|<\delta$, then there exists $v_{1} \in U_{R}$ such that $\rho\left(v_{0}, v_{1}\right)<\varepsilon,\left|f\left(x, v_{1}\right)\right|<\varepsilon$, and $\nabla V(x) \cdot f\left(x, v_{1}\right)<0$.

We conclude this section on Artstein's theorem with the important observation made in [5] that, if a control system is affine in the control, then it is not necessary to enlarge the class of admissible controls to include relaxed controls. In other words, Theorem 2.10 as well as the remark made in the preceding paragraph hold for ordinary feedback controls. A general implication of Artstein's theorem for one-dimensional control systems will be discussed in Section 2.2.3.

\subsection{Stabilisation under controllability assumptions}

The results in Section 2.1 were grouped together on the basis of their applicability to control systems of the general form $\Sigma: \dot{x}=f(x, u)$, assuming only some regularity properties for the map $f$ in order for the relevant constructions to make sense. From the present section onwards we start imposing additional control-theoretic properties on $\Sigma$ and, more specifically, controllability properties, and we consider the implications on the stabilisability of the system. Although a conscious effort is made to maximise the coherence of the presentation as we meander through the literature, the picture becomes necessarily fragmented from now on for the simple reason that there do not exist universal, all-encompassing results that relate the controllability properties of a nonlinear control system to its stabilisability properties. For each result that we present below we specify precisely the class of control systems to which the result applies and the reader should pay attention to the fact that some of those classes of systems are significantly narrower than the one we considered in Section 2.1. ${ }^{7}$

\footnotetext{
${ }^{5} \mathrm{~A}$ semi-metric $\rho$ on a set $X$ is convex if, for any two distinct points $x, y \in X$, there exists a point $z \in X \backslash\{x, y\}$, such that $\rho(x, y)=\rho(x, z)+\rho(z, y)$.

${ }^{6}$ This property is known in the stabilisation literature as the "small-control property".

${ }^{7}$ In Section 2.1 we considered systems of the form $\dot{x}=f(x, u)$, with $f: \mathbb{R}^{n} \times \mathbb{R}^{m} \rightarrow \mathbb{R}^{n}$ merely continuous.
} 
2.2.1. Locally controllable systems. Suppose $M$ is a $C^{r}$-manifold, $r \in\{\infty, \omega\}$, and $\Omega$ a separable metric space. A $C^{r}$-control system on $M$ is a map $f: M \times \Omega \rightarrow T M$ such that, for any $\omega \in \Omega$ fixed, the map $f_{\omega}: M \ni x \mapsto f(x, \omega) \in T M$ is a $C^{r}$-analytic vector field on $M$. If $r=\infty$, we use the designation smooth for the control system and, if $r=\omega$, we use the designation real analytic. The manifold $M$ is usually called the state space of the control system whereas the metric space $\Omega$ can be either the space of control functions or the space of control values depending on the way one wishes to view the control system. If we let $\mathscr{U}_{\text {meas }}$ denote the set of measurable functions $u: I \subset \mathbb{R} \rightarrow \Omega$, defined on an interval $I$ of the real line, then the class of admissible controls for a control system $f$ is defined to be the subset $\mathscr{U}_{f} \subset \mathscr{U}_{\text {meas }}$ containing the functions $u$ with the property that the differential equation $\dot{x}(t)=f(x(t), u(t))$ satisfies Carathéodory's theorem on the existence and uniqueness of solutions. If $p \in M, t \in \mathbb{R}, t \geq 0$, and $t \mapsto x(t ; p, u(t))$ is the trajectory that corresponds to an admissible control $u$ and satisfies $x(0 ; p, u(0))=p$, then the reachable set of $f$ from $p$ at time $t$ is the set

$$
R_{f}(p, t)=\left\{q \in M \mid q=x(t ; p, u) \text { for some } u \in \mathscr{U}_{f}\right\} .
$$

A control system $f: M \times \Omega \rightarrow T M$ is small-time locally controllable (STLC) from a point $p \in M$ if, for every $T>0$, the point $p$ is in the interior of the set

$$
R_{f}(p,[0, T)) \triangleq \bigcup_{t \in[0, T)} R_{f}(p, t)
$$

Unless there is risk for confusion, we refer to STLC systems simply as locally controllable.

We chose to recall the above definitions at the level of generality that is pertinent to the proofs of one of the main results of the thesis in Chapters 3 and 4 . In the next few sections we will specify the different assumptions that need to be made for the results of each section to hold.

One-dimensional control systems. For one-dimensional control systems, a link between controllability and stabilisation is provided by the fact that, for a fairly general class of systems, open-loop controls can, in certain cases and to the same effect, be replaced by closed-loop controls. More precisely, consider the control system $\Sigma: \dot{x}=f(x, u)$, where $f: \mathbb{R} \times U \rightarrow \mathbb{R}$ is a continuous map, and $U \subset \mathbb{R}^{m}$ is a compact and convex set with $0 \in \operatorname{int} U$. Suppose that the class $\mathscr{U}$ of admissible controls consists of the locally integrable maps $u: \mathbb{R} \rightarrow U$ such that, if $z \in \mathbb{R}$, then the negative orbit of $z$ is the set

$$
\mathcal{O}^{-}(z) \triangleq\left\{y \in \mathbb{R} \mid x(T ; y, u(T))=z, \text { for some } u \in \mathscr{U} \text { and } T \in \mathbb{R}_{>0}\right\},
$$

where, as before, $t \mapsto x\left(t ; x_{0}, u(t)\right)$ denotes the trajectory ${ }^{8}$ of $\Sigma$ that corresponds to a control $u \in \mathscr{U}$ and starts at $y \in \mathbb{R}$ at time $t=0$. If we denote by $\mathscr{U}_{\text {pwc }}$ the piecewise constant maps $F: \mathbb{R} \rightarrow U$, then we have the following [23, p. 341].

2.12 Lemma: For all $z \in \mathbb{R}$ and $y \in \mathcal{O}^{-}(z)$, there exist $F \in \mathscr{U}_{\mathrm{pwc}}$ and $T \in \mathbb{R}_{>0}$ such that $x\left(T ; t_{0}, y, F(x(T))\right)=z$.

\footnotetext{
${ }^{8}$ Unique, by assumption.
} 
The elements of $\mathscr{U}_{\text {pwc }}$ will be called piecewise constant controls. If we now assume that $\Sigma$ is real analytic, then, by a theorem due to Grasse [40], local controllability of $\dot{x}=f(x, u)$ is equivalent to local controllability of $\dot{x}=-f(x, u)$ and, therefore, by virtue of Lemma 2.12, we have the following.

2.13 Corollary: If the control system $\Sigma: \dot{x}=f(x, u)$ is real analytic and locally controllable from $p \in \mathbb{R}$, then it can be stabilised to $p$ in finite time using a piecewise constant feedback control.

In [23], Lemma 2.12 is also used in conjunction with the authors' theory of "control sets" to draw conclusions on the asymptotic stabilisation of one-dimensional control systems. However, there are more direct and simpler-to-state characterisations of asymptotic stabilisability for one-dimensional systems that we recall in Section 2.2.3.

Two-dimensional control-affine systems. Consider a control system of the form

$$
\dot{\mathbf{x}}=X(\mathbf{x})+u Y(\mathbf{x}),
$$

where $\mathbf{x}=(x, y) \in \mathbb{R}^{2}, u \in \mathbb{R}$, and $X, Y$ are $C^{\infty}$ vector fields with $X(0)=0$. Because the vector field $X$ vanishes at the origin and the control system (CAS) is assumed to be locally controllable from the origin, it follows that $Y(0) \neq 0$. We can therefore assume, without loss of generality, that (CAS) has the form ${ }^{9}$

$$
\begin{aligned}
& \dot{x}=u, \\
& \dot{y}=f(x, y),
\end{aligned}
$$

where $f \in C^{\infty}\left(\mathbb{R}^{2}\right)$. A general result on the stabilisation of systems of the form (CASr) is the following theorem due to Kawski [53].

2.14 Theorem: If the control system (CASr) is locally controllable from $x=0$, then there exists a Hölder-continuous map $u: \mathbb{R}^{2} \rightarrow \mathbb{R}$ that makes (CASr) locally asymptotically stable at the origin.

The essence of the proof of Theorem 2.14 can be most easily conveyed by means of an example.

2.15 Example: Consider the control system

$$
\begin{aligned}
& \dot{x}=u, \\
& \dot{y}=y-x^{3},
\end{aligned}
$$

of the form $(\mathrm{CASr})$. If $V \in C^{1}\left(\mathbb{R}^{2}\right)$ and

$$
X_{u}(x, y) \triangleq u \frac{\partial}{\partial x}+\left(y-x^{3}\right) \frac{\partial}{\partial y}
$$

then

$$
X_{u} V(x, y)=u \frac{\partial V(x, y)}{\partial x}+\left(y-x^{3}\right) \frac{\partial V(x, y)}{\partial y}
$$

\footnotetext{
${ }^{9}$ For if this is not the case, we can "straighten out" the vector field $Y$ around the origin and then apply a preliminary feedback that will convert (CAS) to (CASr).
} 
We wish to choose $u$ so that $X_{u} V \leq 0$ for the closed-loop system. To this end, we replace the control parameter $u$ with the function

$$
u(x, y)=\frac{\partial V(x, y)}{\partial y}-\frac{\partial V(x, y)}{\partial x}
$$

Then

$$
X_{u} V(x, y)=\frac{\partial V(x, y)}{\partial x} \frac{\partial V(x, y)}{\partial y}-\left(\frac{\partial V(x, y)}{\partial x}\right)^{2}+\left(y-x^{3}\right) \frac{\partial V(x, y)}{\partial y},
$$

and if we set

$$
\frac{\partial V(x, y)}{\partial x}=-\left(y-x^{3}\right)
$$

then the first and the last term in the previous equation cancel out each other to give

$$
X_{u} V(x, y)=-\left(\frac{\partial V(x, y)}{\partial x}\right)^{2} \leq 0
$$

Equation (2.2.3) implies that

$$
V(x, y)=-x y+\frac{x^{4}}{4}+\phi(y)
$$

where $\phi$ is some function of $y$. The problem of stabilising (2.2.3) then reduces to that of choosing $\phi$ so that $V$ becomes positive-definite, for then $V$ would be a Lyapunov function for (2.2.1). It turns out that it is possible to find a suitable $\phi$; for example, some experimentation shows that $\phi(y)=a y^{m}$ with $a=1$ and $m=4 / 3$ makes $V$ into a Lyapunov function for (2.2.1) and (2.2.2) gives the stabilising control.

Although the assumption of local controllability is not used explicitly in the algorithmic procedure described in Example 2.15, it is essential in two ways. First, it guarantees uniqueness of solutions despite the right-hand side of the closed-loop system being only Hölder-continuous and, second, it allows the application of LaSalle's invariance principle to deduce the asymptotic stability from the non-strict inequality satisfied by the derivative of $V$ along the trajectories of the closed-loop system.

The control-affine system in Example 2.9 can be shown to be locally controllable from $0 \in \mathbb{R}^{3}$ and, therefore, shows that Theorem 2.14 does not hold beyond dimension two.

Assuming that $f$ in (CASr) is real analytic, ${ }^{10}$ the following theorem was proven in [30].

2.16 Theorem: The control system (CASr) is continuously asymptotically stabilisable if and only if, for every $\varepsilon>0$, there exist $p=\left(p_{1}, p_{2}\right) \in B^{2}(0 ; \varepsilon)$, with $p_{2}>0$, and $q=$ $\left(q_{1}, q_{2}\right) \in B^{2}(0, \varepsilon)$, with $q_{2}<0$, such that $f(p)<0$ and $f(q)>0$.

The philosophy of the proof of Theorem 2.16 is entirely different from that of Theorem 2.14. Specifically, in [30], a detailed analysis is made, using Puiseux series, of the way the branches of $f$ through the origin separate the plane and a stabilising feedback control is then constructed using partitions of unity. Using again Lyapunov-theoretic arguments, a more straightforward proof of Theorem 2.16 was given in the paper [28] which contains other interesting results on systems of the form (CASr), as well.

\footnotetext{
${ }^{10}$ The paper [30] contains several results, including a sufficient condition for stabilisation when $f$ is in $C^{\infty}\left(\mathbb{R}^{2}\right)$, but not necessarily in $C^{\omega}\left(\mathbb{R}^{2}\right)$.
} 
Time-varying feedback. It must be clear at this point that, for nonlinear control systems, local controllability from a point does not imply local stabilisability, in general, at least not with feedback controls that are continuous in a neighbourhood of that point. Even more, it can be shown that if the trajectories of a closed-loop system are defined in the sense of Filippov, then the control systems that can be stabilised using discontinuous feedback can also be stabilised by means of continuous feedback [25]. In this case, any necessary condition for continuous stabilisability becomes necessary for discontinuous stabilisability in the sense of Filippov as well, and we have seen several examples of control systems that violate such conditions.

One class of feedback controls for which positive conclusions of a fairly general nature can be reached regarding the connection between controllability and stabilisability is the class of time-dependent feedback controls. Not only it can be shown that such feedbacks exist in many (see below for what "many" means) cases but, also, that they enjoy strong regularity properties. The review article [27] can serve as a roadmap for the extensive literature on time-dependent stabilising feedbacks, at least for the most important results that preceded the article's publication. Adhering to the criteria outlined in the introduction to this chapter, we recall in this section only one result on the existence of time-dependent stabilising feedbacks. However, to the best of our knowledge, it is the most general such result.

Consider the control system $\Sigma: \dot{x}=f(x, u)$, where $f \in C^{\omega}\left(\mathbb{R}^{n} \times \mathbb{R}^{m} ; \mathbb{R}^{m}\right)$ with $f(0,0)$. If there exists $t>0$ such that $R_{f}(p, t)$ has non-empty interior, then $\Sigma$ is said to be strongly accessible from $p \in \mathbb{R}^{n}$; it is called locally continuously reachable to the origin in small time if, for any $T>0$, there exist $\varepsilon>0$ and a continuous map $\mathbb{R}^{n} \ni p \mapsto u(p) \in$ $L^{1}\left((0, T) ; \mathbb{R}^{m}\right)$ such that

$$
\lim _{p \rightarrow 0} \sup _{t \in(0, T)}|u(p)(t)|=0
$$

and

$$
x_{0} \in B^{n}(0 ; \varepsilon) \Rightarrow x\left(T ; x_{0}, u\left(x_{0}\right)(T)\right)=0 .
$$

If, for any $T>0$, there exist $\varepsilon>0$ and $u \in C^{0}\left(\mathbb{R}^{n} \times \mathbb{R} ; \mathbb{R}^{m}\right) \cap C^{\infty}\left(\left(\mathbb{R}^{n} \backslash\{0\}\right) \times \mathbb{R} ; \mathbb{R}^{m}\right)$ such that

(i) $u(0, t)=0$, for all $t \in \mathbb{R}$,

(ii) $u(x, t+T)=u(x, t)$, for all $t \in \mathbb{R}$,

(iii) $x(t)=0 \Rightarrow x(s ; x(t), u(x(s), s))=0$, for all $s, t \in \mathbb{R}, s \geq t$, and

(iv) $x(t) \in B^{n}(0, \varepsilon) \Rightarrow x(s)=0$, for all $s, t \in \mathbb{R}, s \geq t+T$,

then the control system $\Sigma$ is said to be locally stabilisable in small time by means of an almost smooth periodic time-varying feedback. The following theorem was proven by Coron [27, Thm 2.23, Thm 2.30].

2.17 Theorem: If the control system $\Sigma: \dot{x}=f(x, u)$ is strongly accessible from $0 \in \mathbb{R}^{n}$, locally continuously reachable to the origin in small time, and if $n \notin\{2,3\}$, then $\Sigma$ is locally stabilisable in small time by means of an almost smooth periodic time-varying feedback. If 
$\Sigma$ is of the form $\dot{x}=\sum_{i=1}^{k} u_{i} g_{i}(x)$, where $k \in \mathbb{Z}_{>0}$ and $g_{i} \in \Gamma^{\omega} T \mathbb{R}^{n}, i=1, \ldots, k$, then the condition $n \notin\{2,3\}$ can be dropped.

The reader is referred again to [27] for applications of Theorem 2.17 to specific systems such as the rotating rigid body with torques as controls and for references to papers that deal with the explicit construction of stabilising time-varying feedbacks.

\subsubsection{Globally controllable systems.}

Real analytic control systems. Sussmann's paper [80] is one of the early works that investigated the relationship between controllability and stabilisability, and contains both a negative and a positive result of a general nature. By means of a counterexample, Sussmann shows that, even if a closed-loop system is understood as a flow which is merely continuous with respect to time and the state variable, a continuous feedback does not always exist. In Sussmann's counterexample, the obstruction to the existence of a continuous stabilising feedback stems from the impossibility of choosing a direction at each point of the state space in a continuous (with respect to the state) manner, and not from the topology of the state space - the latter being $\mathbb{R}^{2}$. Having established the inevitability of utilising discontinuous feedbacks if one is to show a stabilisation result of a substantial level of generality, Sussmann goes on to prove that, for every globally controllable, real analytic control system, there exists a piecewise analytic feedback ${ }^{11}$ that steers every point of the state space to an arbitrary fixed point in finite time. A control system $f: M \times \Omega \rightarrow T M$ is said to be globally controllable if, for every $p, q \in M$, there exists an admissible $u \in \mathscr{U}_{f}$ such that $x(T ; p, u(T))=q$, for some $T \in \mathbb{R}_{\geq 0}$. In [80] it is further assumed that $u$ can be taken to be piecewise constant; a theorem of Grasse [40] guarantees that we can also assume $u$ to be piecewise constant when we show in Chapter 3 that stabilising piecewise analytic feedbacks exist for locally controllable systems as well. To summarise, the following is proven in [80].

2.18 Theorem: Let $f: M \times \Omega \rightarrow T M$ be a real analytic control system that is globally controllable by means of piecewise constant controls. Then, for every $p \in M$, there exists a piecewise analytic feedback that steers every point of $M$ to $p$ in finite time.

The main contribution of the present thesis can be viewed as an extension of Sussmann's result on globally controllable systems, in the sense that the assumption of global controllability is replaced by that of local controllability. These two notions of controllability are related through normal self-reachability [40], but they are not directly comparable in that neither assumption is stronger than the other. Because Sussmann's work provides the foundation for this thesis, most of the definitions and constructions from [80] are included in Chapter 3.

Driftless control systems. In a spirit similar to that of Theorem 2.17, Coron has shown that globally controllable, real analytic, driftless control systems on $\mathbb{R}^{n}$ can be globally asymptotically stabilised using smooth, time-varying, periodic feedback. A driftless con-

\footnotetext{
${ }^{11}$ The precise definition of a piecewise analytic feedback is given in Chapter 3.
} 
trol system on $\mathbb{R}^{n}$ is a control system of the form

$$
\Sigma: \dot{x}=\sum_{i=1}^{m} u_{i} f_{i}(x),
$$

where $u=\left(u_{1}, \ldots, u_{m}\right) \in \mathbb{R}^{m}$ and the $f_{i}, i=1, \ldots, m$, denote vector fields on $\mathbb{R}^{n}$. The regularity of the vector fields $f_{i}$ determines the regularity of $\Sigma$. That is, a real analytic driftless system is a control system of the form (2.2.4) with $f_{i} \in \Gamma^{\omega} T \mathbb{R}^{n}$. The following theorem is proven in [24].

2.19 Theorem: Let $\Sigma$ be a globally controllable, real analytic, driftless control system on $\mathbb{R}^{n}$. Then, for any $T \in \mathbb{R}_{>0}$, there exists $u \in C^{\infty}\left(\mathbb{R}^{n} \times \mathbb{R} ; \mathbb{R}^{m}\right)$ such that

(i) $u(0, t)=0$, for all $t \in \mathbb{R}$,

(ii) $u(x, t+T)=u(x, t)$, for all $(x, t) \in \mathbb{R}^{n} \times \mathbb{R}$, and

(iii) the origin $0 \in \mathbb{R}^{n}$ is a globally asymptotically stable equilibrium of $\Sigma$.

For example, Brockett's integrator (see page 3) can be globally asymptotically stabilised using a smooth, time-varying, periodic feedback $[73] .^{12}$

2.2.3. Asymptotically controllable systems. Probably under the influence of the classical theory of dynamical systems, the stabilisation of control systems has traditionally been (with many exceptions in the recent years, of course) a quest for asymptotically stable closed-loop systems. Therefore, a large part of the literature on the relationship between controllability and stabilisability employs a notion of asymptotic controllability, as opposed to local controllability. Reviewing a representative sample from this body of work is the purpose of the present section.

One-dimensional systems. For one-dimensional, asymptotically controllable systems, we can obtain a result on stabilisability by directly applying Theorem 2.10, as is done in [5]. Moreover, it suffices to assume only attractivity, whereas for higher-dimensional systems, additional assumptions have to be made. The following paragraphs contain the relevant definitions.

Consider a control system $\Sigma: \dot{x}=f(x, u)$, where $x, u \in \mathbb{R}$, and $f: \mathbb{R} \times \mathbb{R} \rightarrow \mathbb{R}$ is locally Lipschitz and satisfies Carathéodory-type conditions for the existence and uniqueness of solutions (see, for example, [76]). Suppose that, for every point $x_{0}$ in a neighbourhood $N$ of $0 \in \mathbb{R}$, there exists a piecewise constant control $u_{x_{0}}$ to which corresponds a unique trajectory $t \mapsto x\left(t ; x_{0}, u_{x_{0}}(t)\right)$ of $\Sigma$ with the property that $\lim _{t \rightarrow+\infty} x\left(t ; x_{0}, u_{x_{0}}(t)\right)=0$. Then $\Sigma$ is said to be attractive ${ }^{13}$ and we have the following [5].

\footnotetext{
${ }^{12}$ Samson's theoretical and applied work are among the earliest that draw attention to time-varying feedbacks.

${ }^{13}$ We refrain from calling $\Sigma$ "asymptotically controllable" because the standard usage of the term entails additional properties which are described in the next section.
} 
2.20 Theorem: If a control system $\Sigma: \dot{x}=f(x, u), x, u \in \mathbb{R}$, is attractive, then there exists a stabilising relaxed feedback.

Proof: Apply Theorem 2.10 with $V(x)=x^{2}$.

Recall that, by definition (page 10), a stabilising relaxed feedback is continuous on $N \backslash\{0\}$. Example 2.6 shows that Theorem 2.20 does not hold in dimensions greater than one.

In Section 2.1.1 we saw a characterisation of feedback stabilisable, one-dimensional control systems; in the same paper [76] where this characterisation is proven, Sontag and Sussmann show that every attractive, one-dimensional system can be stabilised using timevarying feedback. Because stabilising feedbacks that yield a Lipschitz closed loop do not always exist, a definition of a stabilising feedback has to address the problem of defining the trajectories of a closed-loop system. This is the motivation for the following definition of a stabilising feedback given in [76] where the reader can find additional details.

Suppose that, for the control system $\Sigma: \dot{x}=f(x, u)$ above, the class $\mathscr{U}$ of admissible controls consists of the piecewise constant functions defined on $\mathbb{R}_{\geq 0}$ and taking values in $\mathbb{R}$. Then, an asymptotically stabilising feedback for $\Sigma$ is a pair $(K, \hat{u})$ where $K$ is a map from $\mathbb{R} \backslash\{0\}$ to $\mathbb{R}$ and $\hat{u}: \mathbb{R} \backslash\{0\} \ni x_{0} \mapsto u_{x_{0}} \in \mathscr{U}$ is a map such that

(i) $t \mapsto x\left(t ; x_{0}, u_{x_{0}}(t)\right)$ is defined for all $t \geq 0$,

(ii) $\lim _{t \rightarrow+\infty} x\left(t ; x_{0}, u_{x_{0}}(t)\right)=0$, and

(iii) $\hat{u}_{x\left(t ; x_{0}, u_{x_{0}}(t)\right)}(s)=K\left(x\left(t+s ; x_{0}, u_{x_{0}}(t+s)\right)\right)$, for all $t \geq 0$ and almost all $s \geq 0$.

A time-varying asymptotically stabilising feedback is defined similarly, but with $K$ : $\mathbb{R} \backslash\{0\} \times \mathbb{R}_{\geq 0} \rightarrow \mathbb{R}$. We can now state the following result.

2.21 Theorem: If a control system $\Sigma: \dot{x}=f(x, u), x, u \in \mathbb{R}$, is attractive, then there exists a continuous time-varying asymptotically stabilising feedback.

2.22 Remark: The proof of Theorem 2.21 actually yields a feedback such that the map $x \mapsto f(x, K(x, t))$ is locally Lipschitz and, as the authors point out, it can be modified to yield a map $K$ which is smooth in $x$, for every $x \in \mathbb{R} \backslash\{0\}$.

The example in the Appendix of [80] (which is too long to reproduce here) shows that Theorem 2.21 does not hold in dimensions greater than one.

Lyapunov characterisation of asymptotic controllability. The control systems of the previous section were of the general form $\dot{x}=f(x, u)$. However their state space was restricted to be one-dimensional. In the case where the state space is $\mathbb{R}^{n}, n \geq 2$, stabilisation results of a fairly general nature have also been proven, however the distinctive feature between the cases $n=1$ and $n \geq 2$ is that, in general, "regular" (e.g., continuous) stabilising feedbacks do not exist in the latter case. Because of this inherent lack of regularity, part of every contribution in this area has been to provide a meaningful definition for the trajectories of a closed-loop system. Two ideas that have prevailed and have been generalised in several directions since their appearance are the Lyapunov characterisation of asymptotic controllability, due to Sontag [77], and the patching of open-loop controls into a closed-loop 
system, due to Ancona and Bressan [3]. This section and the next one describe these two ideas in more detail.

To state the main result in [77] — that is, the equivalence between asymptotic controllability and the existence of a control-Lyapunov function - we need the following definitions. Consider the control system $\Sigma: \dot{x}=f(x, u)$, where $x \in \mathbb{R}^{n}, t \in \mathbb{R}_{\geq 0}$, and assume that the class of admissible controls is

$$
\mathscr{U} \triangleq\left\{u: \mathbb{R}_{\geq 0} \rightarrow \mathbb{R}^{m} \mid u \text { is measurable and locally essentially bounded }\right\} \text {. }
$$

Suppose that the map $f: \mathbb{R}^{n} \times \mathbb{R}^{m} \rightarrow \mathbb{R}^{n}$ is locally Lipschitz, that the point $(0,0) \in \mathbb{R}^{n} \times \mathbb{R}^{m}$ is an equilibrium, that is, $f(0,0)=0$, and that, for any admissible control $t \mapsto u(t)$, there exists locally a unique solution of $\dot{x}(t)=f(x(t), u(t))$. The control system $\Sigma$ is said to be asymptotically controllable to $0 \in \mathbb{R}^{n}$ if

(i) for any $x_{0} \in \mathbb{R}^{n}$ there exists an admissible control $u \in \mathscr{U}$ such that $\lim _{t \rightarrow+\infty} x\left(t ; x_{0}, u(t)\right)=0$

(ii) for any $\varepsilon>0$ there exists a $\delta>0$ and an admissible control $u \in \mathscr{U}$ such that $\left|x_{0}\right|<\delta$ implies $\lim _{t \rightarrow+\infty} x\left(t ; x_{0}, u(t)\right)=0$ and $\left|x\left(t ; x_{0}, u(t)\right)\right|<\varepsilon$, for all $t \geq 0$, and

(iii) there exist $\eta, k \in \mathbb{R}_{>0}$ such that $\left|x_{0}\right|<\eta$ implies that the control in (ii) can be chosen so that $|u|<k$.

In order to prove the existence of a control-Lyapunov function for asymptotically controllable systems, it is necessary to consider relaxed controls in addition to the classical controls. In Section 2.1.4 the focus was on feedback relaxed controls; in the present section we need the following more general definition of an open-loop relaxed control (see also the footnote on page 10).

Let $W$ denote the set of probability measures on $\mathbb{R}^{m}$ equipped with the vague topology ${ }^{14}$ and let $W_{r} \subset W$ denote the subspace of probability measures supported in $B(0, r)$. A relaxed control is a measurable function $w: \mathbb{R}_{\geq 0} \rightarrow W$. The class of relaxed controls is denoted by $\mathscr{W}$ and the class of relaxed controls taking values in $W_{r}$ for almost every $t$ is denoted by $\mathscr{W}_{r}$. A control-Lyapunov function is a positive-definite, continuous, proper function $V: \mathbb{R}^{n} \rightarrow \mathbb{R}$ such that, for every $x \in \mathbb{R}^{n}$, there exists $w \in \mathscr{W}$ such that

$$
\dot{V}_{w}(x) \triangleq \liminf _{t \rightarrow 0^{+}} \frac{V(x(t ; x, w))-V(x)}{t}<0 .
$$

As stated at the beginning of this section, the following theorem is proven in [77].

2.23 Theorem: The control system $\Sigma: \dot{x}=f(x, u)$ is asymptotically controllable if and only if there exists a control-Lyapunov function.

With a smooth control-Lyapunov function at hand, the construction of a stabilising feedback is straightforward. However, the control-Lyapunov function of Theorem 2.23 is Lipschitz continuous ${ }^{15}$ and there are technical difficulties to overcome if it is to be used

\footnotetext{
${ }^{14}$ See [31, Ch. XIII, §4], for example.

${ }^{15}$ Many of the examples we have seen so far show that asymptotic controllability does not imply the existence of a smooth control-Lyapunov function.
} 
for the construction of a stabilising feedback. This task is undertaken in [22] where it is shown that asymptotic controllability implies the existence of a stabilising feedback. The philosophy of the proof is to obtain a stabilising feedback as the solution to a sequence of optimisation problems that are initialised by sampling the state of the control system. To state the result in [22] a few more definitions are necessary.

Define a feedback to be a function $k: \mathbb{R}^{n} \rightarrow \mathbb{R}^{m}$ that maps compact sets to relatively compact sets and a partition of $[0, \infty)$ to be an infinite increasing sequence $\pi=\left(t_{i}\right)_{i \geq 0}$ with $t_{0}=0$. The number $d(\pi)=\sup _{i}\left(t_{i+1}-t_{i}\right)$ is called the diameter of $\pi$. Given a feedback $k$, a partition $\pi$, and an initial state $x_{0} \in \mathbb{R}^{n}$, the $\pi$-trajectory of $\Sigma$ starting at $x_{0}$ is the trajectory obtained by solving recursively the differential equations

$$
\dot{x}(t)=f\left(x(t), k\left(x\left(t_{i}\right)\right)\right), \quad t \in\left[t_{i}, t_{i+1}\right] .
$$

A feedback $k$ is said to $s$-stabilise $\Sigma$ if, for any two positive real numbers $R$ and $r$ with $R>r$, there exist $M_{R}>0, \delta_{R, r}>0$, and $T_{R, r}>0$ such that, for every partition $\pi$ of $[0, \infty)$ with $d(\pi)<\delta$ and every initial state $x_{0}$ with $\left|x_{0}\right| \leq R$, the $\pi$-trajectory $\gamma$ of $\dot{x}=f(x, k(x))$ starting at $x_{0}$ is well-defined and satisfies:

(i) $|\gamma(t)| \leq r$, for all $t \geq T$;

(ii) $|\gamma(t)| \leq M$, for all $t \geq 0$; and

(iii) $\lim _{R \rightarrow 0} M(R)=0$.

The following is proven in [22].

2.24 Theorem: A system $\Sigma: \dot{x}=f(x, u)$ is asymptotically controllable if and only if there exists an s-stabilising feedback.

As the authors point out, the feedback of Theorem 2.24 is not robust with respect to state measurement errors. Subsequent work [59, 69], however, improved on this aspect of the result.

Patchy feedback. Another approach to the stabilisation of asymptotically controllable systems in $\mathbb{R}^{n}$ is a more direct construction of a stabilising feedback by patching together the open-loop controls that drive the states of a system to the origin. Such feedbacks are called "patchy" in [3], where their existence is proven. The result of [3] is stated below after the necessary definitions are given; it applies to systems of the form $\Sigma: \dot{x}=f(x, u)$, where $x \in \mathbb{R}^{n}, u \in K \subset \mathbb{R}^{m}$, with $K$ compact, and the map $f: \mathbb{R}^{n} \times \mathbb{R}^{m} \rightarrow \mathbb{R}^{n}$ is smooth.

A patch is a pair $(\Omega, g)$ where $\Omega \subset \mathbb{R}^{n}$ is an open domain with smooth boundary and $g$ is smooth vector field defined on a neighbourhood of $\bar{\Omega}$ and satisfying

$$
\langle g(x), n(x)\rangle<0, \quad \forall x \in \partial \Omega .
$$

The vector $n(x)$ is the unit normal at $x \in \partial \Omega$ pointing outside $\Omega$. A patchy vector field is a triple $\left(\Omega, g,\left(\Omega_{a}, g_{a}\right)_{a \in A}\right)$ such that

(i) the index set $A$ is totally ordered,

(ii) $\Omega$ and $\Omega_{a}, a \in A$, are open domains with smooth boundary, 
(iii) $\left\{\Omega_{a}\right\}_{a \in A}$ is a locally finite cover of $\Omega$,

(iv) $g: \Omega \rightarrow \mathbb{R}^{n}$ is a map and $\left\{\left(\Omega_{a}, g_{a}\right)\right\}_{a \in A}$ is a family of patches such that

$$
g(x)=g_{a}(x), \text { whenever } x \in \Omega_{a} \backslash \bigcup_{b>a} \Omega_{b}
$$

Given a patchy vector field $\left(\Omega, g,\left(\Omega_{a}, g_{a}\right)_{a \in A}\right)$, if there exist control values $k_{a} \in K, a \in A$, such that

$$
g_{a}(x)=f\left(x, k_{a}\right), \quad \forall x \in \Omega_{a} \backslash \bigcup_{b>a} \Omega_{b},
$$

then the piecewise constant map

$$
U(x)=k_{a}, \quad \forall x \in \Omega_{a} \backslash \bigcup_{b>a} \Omega_{b}
$$

is called a patchy feedback on $\Omega$. Patchy feedbacks have piecewise $C^{1}$ integral curves and the following theorem says that they exist for asymptotically controllable systems [3]. For the result to hold, the last part in the definition of asymptotic controllability, that is, the small-control property, is not necessary.

2.25 Theorem: If a system $\Sigma: \dot{x}=f(x, u)$ is asymptotically controllable, then there exists an asymptotically stabilising patchy feedback.

The patchy feedback of Theorem 2.25 is shown to be robust with respect to certain types of disturbances and a more extensive analysis of robustness properties is presented in [4]. Theorem 2.25 is used in [39] to prove the existence of piecewise smooth, patchy, control-Lyapunov functions for asymptotically controllable systems. Other papers that contain recent results of a general nature on the existence of stabilising feedbacks and on Lyapunov-like characterisations of control-theoretic properties are [54, 66, 70, 71, 84]. 


\section{Chapter 3}

\section{Feedback stabilisation of locally controllable systems}

\subsection{Introduction}

The interest in studying semianalytic sets, i.e., sets that locally satisfy finitely many analytic inequalities, is self-evident since many important families of sets in mathematics and its applications are semianalytic, e.g., polyhedra in $\mathbb{R}^{n}$. It is thus not surprising that they have been studied extensively, beginning with the work of Thom and Lojasiewicz who established their fundamental properties such as the existence of stratifications and triangulations [74]. However, the image of even a compact semianalytic set under an analytic map can fail to be semianalytic [45] and, therefore, the class of semianalytic sets has to be enlarged in order for their properties to be studied effectively. The solution was given independently by Gabrielov [35], Hardt [45], and Hironaka [47, 48], who introduced the category of subanalytic sets. We should note here that, although the study of semianalytic sets naturally led to the concept of a subanalytic set, the applicability of subanalytic sets extends well beyond the analysis of semianalytic sets.

The theory of subanalytic sets was used for the first time in control theory by Brunovský [16] to show the existence of a regular time-optimal synthesis [14] for linear systems with constraints. At the same time, the theory of subanalytic sets was used by Sussmann to show in [79] the existence of universal inputs (a problem related to nonlinear observability) and in [80] the existence of stabilising feedbacks for nonlinear globally controllable systems.

\subsection{Real analytic geometry}

The goal of this section is to introduce a minimal background from real analytic geometry and, more specifically, from the theory of subanalytic sets. Starting from the definitions of semianalytic and subanalytic sets, we introduce the parts of the theory that are necessary in order to state a stratification theorem due to Sussmann [80] which is the cornerstone of the construction of a piecewise analytic feedback. Unless explicitly stated otherwise, all manifolds that appear in this chapter and the next one are, by definition, second-countable, Hausdorff, topological spaces, equipped with a maximal real analytic atlas. 
3.2.1. Semi-analytic and subanalytic sets. Let $U$ be an open subset of a manifold $M$ and denote the ring of real analytic functions defined on $U$ by $C^{\omega}(U ; \mathbb{R})$. To make the length and complexity of certain statements manageable, we introduce the following notation. If $f: A \rightarrow \mathbb{R}$ is a function on a set $A$ and $\sigma \in\{=,>\}$, then

$$
\{f \sigma 0\} \triangleq\{a \in A \mid f(a) \sigma 0\} .
$$

Moreover, we set

$\mathcal{S}\left(C^{\omega}(U ; \mathbb{R})\right) \triangleq\left\{X \subset U \mid X=\bigcup_{i=1}^{p} \bigcap_{j=1}^{q}\left\{f_{i j} \sigma 0\right\}, f_{i j} \in C^{\omega}(U ; \mathbb{R}), \sigma \in\{=,>\}, p, q \in \mathbb{Z}_{>0}\right\}$.

Then, a subset $X$ of a manifold $M$ is said to be semianalytic if, for every $p \in M$, there exists a neighbourhood $U$ of $p$ in $M$ such that $X \cap U \in \mathcal{S}\left(C^{\omega}(U ; \mathbb{R})\right)$. The study of semianalytic sets leads naturally to the notion of subanalytic sets mainly because the class of semianalytic sets is not closed under projections, and subanalytic sets are precisely projections of (compact) semianalytic sets. Specifically, a subset $X$ of a manifold $M$ is subanalytic if, for every point $p \in M$, there exists a neighbourhood $U$ of $p$ in $M$, a manifold $N$, and a compact semianalytic subset $A$ such that $X \cap U=\operatorname{pr}_{1}(A)$, where $\operatorname{pr}_{1}: M \times N \rightarrow M$ is the canonical projection onto the first factor. This definition of subanalytic sets is the first of the three equivalent definitions given in [13, Prop. 3.13]; the second part of the same Proposition is the definition that appears in [80].

3.2.2. Stratifications. One of the most useful tools the theory of subanalytic sets provides for applications is the stratification theorems for locally finite families of subanalytic sets [58, $79,80,83]$. In what follows, we recall the definition of a subanalytic stratifiaction, following the exposition in [80]. The theorems of the following section, although independent from control-theoretic considerations, will take us one step closer to geometric control theory. In particular, if one thinks of a control system as a collection of vector fields on a manifold, the usefulness of stratification theorems for proving existence theorems, e.g., existence of a stabilising feedback, will become apparent.

An analytic stratification of a manifold $M$ is a partition $\mathscr{P}$ of $M$ into connected real analytic submanifolds, called strata, such that

(i) $\mathscr{P}$ is locally finite; that is, for every point $p \in M$, there exists a neighbourhood $U$ of $p$ in $M$ such that $U$ intersects only finitely many strata from $\mathscr{P}$,

(ii) $\bar{S}=\bigcup_{\substack{T \in \mathscr{P} \\ T \cap \bar{S} \neq \emptyset}} T$, for every $S \in \mathscr{P}$; that is, the closure $\bar{S}$ of a stratum $S$ is the union of those stata that have nonempty intersection with $\bar{S}$, and

(iii) $(T \subset \bar{S}) \wedge(T \neq S) \Rightarrow \operatorname{codim} T>\operatorname{codim} S$; that is, if a stratum $T$ is contained in the closure $\bar{S}$ and it is not the whole stratum $S$, then the codimension ${ }^{1}$ of $T$ is larger than the codimension of $S$.

\footnotetext{
${ }^{1}$ A point $x$ of a subanalytic subset $X$ of a manifold $M$ is called smooth of dimension $k$ if there exists a neighbourhood $U$ of $x$ in $M$ such that $X \cap U$ is an analytic submanifold of $M$ of dimension $k$. The dimension of $X$ is defined to be the maximum of the dimensions of the smooth points of $X$.
} 
A subanalytic stratification is an analytic stratification whose strata are subanalytic sets. A stratification is called compatible with a family $\mathscr{A}$ of subsets of $M$ if every $\mathscr{A}$ is a union of strata. As mentioned earlier, the next theorem is fundamental for showing that a piecewise analytic feedback, with all its defining properties, exists for locally or globally controllable systems.

3.1 Theorem: [80] Let $\mathscr{A}$ be a locally finite family of nonempty subanalytic subsets of $M$ and, for each $A \in \mathscr{A}$, let $F(A)$ be a finite collection of vector fields on $M$. Then there exists a subanalytic stratification $\mathscr{P}$ of $M$ compatible with $\mathscr{A}$ and having the following property: whenever $\mathscr{P} \ni S \subset A \in \mathscr{A}$ and $X \in F(A)$, then either $X$ is everywhere tangent to $S$ or $X$ is nowhere tangent to $S$.

\subsection{Control theory}

In the remainder of this chapter, and unless specified otherwise, all control systems are assumed to be real analytic. The reader may need to refer back to previous sections, and Section 2.2.1 in particular, for notation and definitions that are used below.

3.3.1. Piecewise analytic feedback. The first point of contact between the real analytic geometry of the previous section and control theory is the notion of a piecewise analytic feedback. That is, a piecewise analytic vector field that can be realised by choosing values for the controls of a control system. We begin with the definition of a piecewise analytic vector field [80].

A piecewise analytic vector field on a manifold $M$ is a quadruple

$$
V=\left(\Sigma,\left(\Sigma_{1}, \Sigma_{2}\right),\left\{V_{S}\right\}_{S \in \Sigma_{1}}, E\right),
$$

where

(i) $\Sigma$ is an analytic stratification of $M$,

(ii) $\left(\Sigma_{1}, \Sigma_{2}\right)$ is a partition of $\Sigma$; strata in $\Sigma_{1}$ are said to be of the first kind and strata in $\Sigma_{2}$ of the second kind,

(iii) for every $S \in \Sigma_{1}, V_{S}$ is an analytic vector field on $S$,

(iv) for every $p \in S \in \Sigma_{1}$, the integral curve $\gamma$ of $V_{S}$ through $p$ is either defined for all $t \geq 0$ or, if the integral curve is defined up to some time $T>0$, then $\lim _{t \rightarrow T^{-}} \gamma(t)$ exists,

(v) $E$ is a map which assigns to every point $p$ in a stratum $S \in \Sigma_{2}$ a stratum $E(p) \in \Sigma_{1}$,

(vi) for every $p \in S \in \Sigma_{2}$, there exists a unique integral curve $\gamma$ of $V_{E(p)}$ such that $\lim _{t \rightarrow 0^{+}} \gamma(t)=p$.

A piecewise analytic feedback for a control system $f: M \times \Omega \rightarrow T M$ can now be defined as a piecewise analytic vector field $V$ on $M$ such that, for every $p \in S \in \Sigma_{1}$, there exists $\omega \in \Omega$, such that $V_{S}(p)=f(p, \omega)$.

If $p \in S \in \Sigma_{2}$, that is, $p$ belongs to a stratum of the second kind, then the map $E$ in the definition of a piecewise analytic vector field is used as an "exit rule" from $S$ into $E(p) \in \Sigma_{1}$. 
3.3.2. Normal reachability. This section is concerned with normal reachability, a property of real analytic, locally controllable systems that is essential for proving the existence of a piecewise analytic feedback. In geometric control theory, there is an obvious appeal in knowing that certain control-theoretic tasks can be accomplished using piecewise constant controls. This is due on the one hand to the simplicity of such controls and, on the other, to the direct geometric interpretation of a control system with piecewise constant controls as a family of vector fields on a manifold. For the purpose of constructing a piecewise analytic feedback, we need not only know that the points in the reachable set of a locally controllable system can be reached using piecewise constant controls, but also that the concatenation of flows that correspond to these piecewise constant controls have rank equal to the dimension of the state space, as a map from the space of switching times to the state space of the control system. This last property is called normal reachability and it has been shown to be true for a wide class of control systems [40]. Normal reachability entails the use of piecewise constant controls and, therefore, we have to make sure that such controls are admissible. To this end, we assume that $T f_{\omega}: T M \times \Omega \rightarrow T T M^{2}$ is continuous. Then $\mathscr{U}_{f}$ contains the set $\mathscr{U}_{\text {step }}$ of piecewise constant maps that possess a finite number of discontinuities on every bounded interval $[40,41]$ [78, p. 474 Thm 54, p. 480 Prop. C3.4].

If $\Phi^{f}: \mathbb{R} \times M \supset U \ni(t, p) \mapsto \Phi^{f}(t, p) \in M$ is the flow (defined on some open subset $U$ ) of a vector field $f$ on a manifold $M$, we write $\Phi_{p}^{f}$ for the map $t \mapsto \Phi^{f}(t, p)$ [18, p. 95]. With this notation, given a control system $f: M \times \Omega \rightarrow T M$ and two points $p, q \in M$, we say that $q$ is normally reachable from $p$ (via $f$ ) if there exist $k \in \mathbb{N},\left(\omega_{1}, \ldots, \omega_{k}\right) \in \Omega^{k}$, and

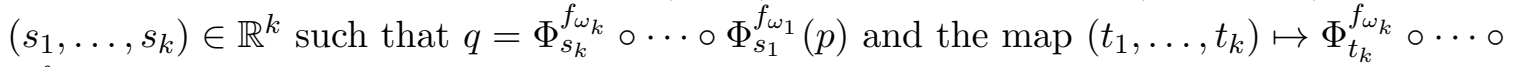
$\Phi_{t_{1}}^{f_{\omega_{1}}}(p)$ has rank equal to $\operatorname{dim} M$ at $\left(s_{1}, \ldots, s_{k}\right)$.

The following theorem is a combination of results contained in [40] and it covers all aspects of normal reachability that are relevant to the proof of stabilisability of locally controllable systems given in Section 3.4 below.

3.2 Theorem: If a control system $f: M \times \Omega \rightarrow T M$ is locally controllable from $p \in M$, then the time-reversed system $-f$ is also locally controllable from $p$ and every point in $R_{-f}(p,[0, T))$ is normally reachable from $p$.

As stated earlier, the theorem guarantees that we can actually reach an open neighbourhood of each point in the reachable set of a locally controllable system.

\subsection{Proof of the main result}

Given a control system $f: M \times \Omega \rightarrow T M$, a point $p \in M$ is said to be an equilibrium for $f$ if there exists $\omega \in \Omega$ such that $f(p, \omega)=0$. The control system $f$ is said to admit a uniform bound at $p$ if, given a positive real number $T$, there exists a compact set containing all trajectories defined on $[0, T]$ and starting at $p[60]$.

3.3 Theorem: Let $f: M \times \Omega \rightarrow T M$ be a control system locally controllable from an equilibrium $p \in M$. Suppose $f$ admits a uniform bound, $\Omega$ is compact, and $\{f(p, \omega) \mid \omega \in \Omega\}$ is convex for every $p \in M$. There exists a locally asymptotically stabilising piecewise analytic feedback.

\footnotetext{
${ }^{2}$ Fix $\omega$ to compute $T f_{\omega}$ and consider the resulting map on $T M \times \Omega$.
} 
Proof: If $\xi=\left(X_{1}, \ldots, X_{k}\right)$ is a finite sequence of vector fields, $|\xi|$ denotes the number of elements in $\xi$, i.e., $|\xi|=k$, and if $\tau=\left(t_{1}, \ldots, t_{k}\right)$ is a $k$-tuple of real numbers, $|\tau|$ is equal to the sum of the components of $\tau$, i.e. $|\tau|=t_{1}+\cdots+t_{k}$. Given such a $\tau \in \mathbb{R}^{k}$, we denote the cubical neighbourhood of $\tau$ of side $2 \varepsilon$ by $C_{\varepsilon}^{n}(\tau)$; in other words,

$$
C_{\varepsilon}^{k}(\tau)=\left\{\left(s_{1}, \ldots, s_{k}\right) \in \mathbb{R}^{k}|| t_{i}-s_{i} \mid \leq \varepsilon, i \in\{1, \ldots, n\}\right\} .
$$

The non-negative orthant of $\mathbb{R}^{k}$ will be denoted by $\mathbb{R}_{\geq 0}^{k}$ and it is, by definition, the set $\left\{\tau=\left(t_{1}, \ldots, t_{k}\right) \in \mathbb{R}^{k} \mid t_{i} \geq 0, i \in\{1, \ldots, k\}\right\}$. For a collection of vector fields $\xi$ and a $k$-tuple $\tau$ as above, $\Phi_{\tau}^{\xi}$ stands for the composition $\Phi_{t_{1}}^{X_{1}} \circ \cdots \circ \Phi_{t_{k}}^{X_{k}}$ and, similarly to the case of a single vector field, $\Phi_{p}^{\xi}$ is the map $\tau \mapsto \Phi^{\xi}(\tau, p)$.

Since the control system $f$ is locally controllable from $p$, the time-reversed system $-f$ is also locally controllable from $p$ by Theorem 3.2. By the same theorem, if $q$ is a point in the reachable set $R_{-f}(p,[0, T])$ of $-f$, then $q$ is normally reachable from $p$ via $-f$ in time less than $T+\alpha$, where $\alpha$ is a positive real number. That this is the case follows from the fact that $R_{-f}(p,[0, T]) \subset R_{-f}(p,[0, T+\alpha))$. Theorem 3.2 can then be applied directly to the set $R_{-f}(p,[0, T+\alpha))$. Normal reachability of $q$ from $p$ via $-f$ in time less than $T+\alpha$ means precisely that we can find a finite sequence of vector fields $\xi_{q}=\left(X_{1}, \ldots, X_{\left|\xi_{q}\right|}\right)$ and $\sigma_{q}=\left(s_{1}, \ldots, s_{\left|\xi_{q}\right|}\right) \in \mathbb{R}_{\geq 0}^{\left|\xi_{q}\right|}$ such that $\left|\sigma_{q}\right|<T+\alpha$, each $X_{i}, i \in\left\{1, \ldots,\left|\xi_{q}\right|\right\}$, is of the form $-f_{\omega}$, and $\Phi^{\xi_{q}}\left(\sigma_{q}, p\right) \triangleq \Phi_{s_{1}}^{X_{1}} \circ \cdots \circ \Phi_{s_{\left|\xi_{q}\right|}}^{X_{\left|\xi_{q}\right|}}(p)=q$, with the map $\Phi_{p}^{\xi_{q}}$ having rank equal to the dimension of $M$ at $\sigma_{q}$. Since the rank of the map $\Phi_{p}^{\xi_{q}}$ is $\operatorname{dim} M$ at $\sigma_{q}$, the set $\Phi_{p}^{\xi_{q}}\left(C_{\varepsilon_{q}}^{\left|\xi_{q}\right|}\left(\sigma_{q}\right) \cap \mathbb{R}_{\geq 0}^{\left|\xi_{q}\right|}\right)$ contains a neighbourhood of $q$, for some (in fact, any) positive $\varepsilon_{q}$. For every $\tau \in C_{\varepsilon_{q}}^{\left|\xi_{q}\right|}\left(\sigma_{q}\right) \cap \mathbb{R}_{\geq 0}^{\left|\xi_{q}\right|}$, we can define a curve

$$
\eta_{\tau}:[0,|\tau|] \ni t \mapsto \eta_{\tau}(t) \in \mathbb{R}^{\left|\xi_{q}\right|}
$$

such that $t \mapsto\left(\Phi_{p}^{\xi_{q}} \circ \eta_{\tau}\right)(t)$ is the concatenation of trajectories of the control system $-f$ that connects $p$ and $\Phi_{p}^{\xi_{q}}(\tau)$ (see Figure 3.1). Let $A_{q}$ denote the set of points in $\mathbb{R}_{\geq 0}^{\left|\xi_{q}\right|}$ which are of the form $\eta_{\tau}(t), \tau \in C_{\varepsilon_{q}}^{\left|\xi_{q}\right|}\left(\sigma_{q}\right) \cap \mathbb{R}_{\geq 0}^{\left|\xi_{q}\right|}, t \in[0,|\tau|]$. The set $A_{q}$ can be written as a finite union of compact semianalytic sets: $A_{q}=A_{q}^{1} \cup \cdots \cup A_{q}^{\left|\xi_{q}\right|}$. The geometric meaning of the $A_{q}^{i}$ is shown in Figure 3.2; they are sets of increasing dimension and every $A_{q}^{i}$ is a rectangular "neighbourhood" of the $i$ th segment of the curve $\eta_{\tau}$. The explicit description of the sets $A_{q}^{i}$ in terms of inequalities - inequalities that stem form the fact that $\tau \in C_{\varepsilon_{q}}^{\left|\xi_{q}\right|}\left(\sigma_{q}\right) \cap \mathbb{R}_{\geq 0}^{\left|\xi_{q}\right|}$ and $t \in[0,|\tau|]$ - is as follows [80, p. 45]: the set $A_{q}^{i}$ consists of those points $\left(t_{1}, \ldots, t_{\left|\xi_{q}\right|}\right)$ that satisfy

(i) $t_{j}=0$, for $j \leq\left|\xi_{q}\right|-i$,

(ii) $0 \leq t_{j} \leq b_{j}$, for $j=\left|\xi_{q}\right|+1-i$, and

(iii) $a_{j} \leq t_{j} \leq b_{j}$, for $j>\left|\xi_{q}\right|+1-i$,

where $a_{q}^{i}=\max \left(\sigma_{q, i}-\varepsilon_{q}, 0\right), b_{q}^{i}=\sigma_{q, i}+\varepsilon_{q}$, and $\sigma_{q, i}$ denotes the $i$ th component of $\sigma_{q}$. Since the sets $A_{q}, A_{q}^{i}$ are compact semianalytic, their images $B_{q}=\Phi_{p}^{\xi_{q}}\left(A_{q}\right), B_{q}^{i}=\Phi_{p}^{\xi_{q}}\left(A_{q}^{i}\right)$ are compact subanalytic sets. The set $B_{q}$ contains a neighbourhood of the point $q$. 


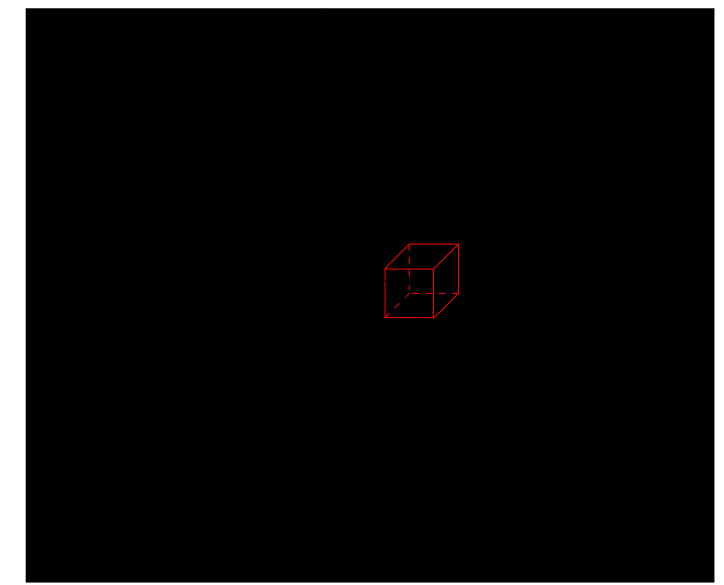

Figure 3.1. The curve $\eta_{\tau}$ is used to express a concatenation of flows for a control system as one single curve.

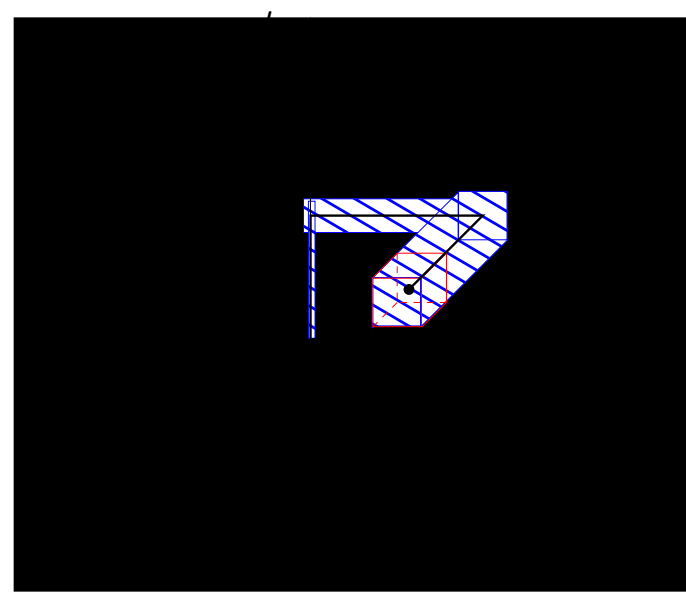

Figure 3.2. A graphical representation of the sets $A_{q}^{i}$ used in the proof of Theorem 3.3.

Consider now a strictly decreasing sequence $\left(T_{i}\right)$ of positive real numbers with $T_{i} \rightarrow 0$ and $T_{1}=T$. The assumptions of the theorem imply that the sets $R_{-f}\left(p,\left[0, T_{i}\right]\right)$ form a decreasing sequence of compact sets [60, p.242]. For the ease of notation, set $K_{i}=$ $R_{-f}\left(p,\left[0, T_{i}\right]\right)$ and $\stackrel{\circ}{K}_{i}=R_{-f}\left(p,\left[0, T_{i}\right)\right)$. Each point $q$ in $R_{i} \triangleq K_{i} \backslash \operatorname{int} K_{i+1}$ is contained in a set $B_{q}$ and, because $R_{i}$ is compact, every $R_{i}$ can be covered with finitely many sets $B_{q_{1}}, \ldots, B_{q_{k_{i}}}$. We reindex the points $q_{i}$ that correspond to the sets that form the finite covers to create a sequence $B_{q_{1}}, B_{q_{2}}, \ldots$ of sets. Using the indices $i, j$, and $m$ uniquely defined by the relation

$$
j=i+\left|\xi_{q_{1}}\right|+\cdots+\left|\xi_{q_{m-1}}\right|,
$$

with $1 \leq i \leq\left|\xi_{q_{m}}\right|$, we define the sequence of sets $D_{j}=B_{q_{m}}^{\left|\xi_{q_{m}}\right|+1-i}$ and we set $H_{j}=$ 
$D_{j} \backslash \bigcup_{i \neq j} D_{i}$. The sequence $\left(H_{j}\right)_{j \geq 1}$ gives, by restriction of certain sets $H_{j}$ if necessary, a locally finite partition of the punctured neighbourhood $\stackrel{\circ}{K_{1}} \backslash\{p\}$ of $p$ into relatively compact subanalytic sets. To each set $H_{j}, j \geq 1$, we assign the vector field $Y_{j}=-X_{i}$, where $i$ and $j$ satisfy (3.4.1), and to $H_{0} \triangleq\{p\}$ we assign the zero vector field; each vector field $X_{i}$ is of the form $-f_{\omega}$ and, therefore, $Y_{j}$ is of the form $f_{\omega}$. Applying Theorem 3.1 to the family $\mathscr{H}=\left\{H_{j}\right\}_{j \geq 1}$ of subanalytic subsets, with $F\left(H_{j}\right)=\left\{Y_{j}\right\}$ (see the notation in the theorem), gives a stratification $\Sigma$ of $\stackrel{\circ}{K}_{1}$, compatible with the family $\mathscr{H}$. The stratification $\Sigma$ is partitioned into $\Sigma_{1}$ and $\Sigma_{2}$ in the following way: every stratum $S \in \Sigma$ is a subset of an $H_{j}$; if $Y_{j}$ is everywhere tangent to $S$ then $S \in \Sigma_{1}$, otherwise $S \in \Sigma_{2}$.

We now show that the trajectories of the piecewise analytic feedback starting in a neighbourhood of $p$ converge to $p$. More specifically, let $q$ be an arbitrary point in $\stackrel{\circ}{K_{1}} \backslash\{p\}$; then $q$ belongs to $H_{j}$, for some $j>0$, since $\left(H_{j}\right)_{j \geq 1}$ is a locally finite partition of $\stackrel{\circ}{K_{1}} \backslash\{p\}$. By definition of the set $H_{j}, q$ belongs to $D_{j}$ but not to $E_{j-1}$ and, therefore, $q$ is a point of the form

$$
q=\Phi_{t_{i}^{0}}^{X_{i}} \circ \cdots \circ \Phi_{t_{k}^{0}}^{k}(p),
$$

for some finite sequence $\left(t_{i}^{0}, \ldots, t_{k}^{0}\right)$ of times. The curve

$$
\gamma: s \rightarrow \Phi_{t_{i}^{0}-s}^{X_{i}} \circ \cdots \circ \Phi_{t_{k}^{0}}^{X_{k}}
$$

is the integral curve of $-X_{i}$ that satisfies $\gamma(0)=q$. For $s=t_{i}^{0}$ we have $\gamma\left(t_{i}^{0}\right) \in E_{j+1}$ and, since $Y_{j}=-X_{i}$, the trajectory of the piecewise analytic feedback that starts at $q$ successively enters sets $H_{j}$ with increasing index $j$ approaching, thus, the point $p$.

Recall that an equilibrium $p$ is Lyapunov stable if, for any neighbourhood $U$ of $p$, there exists a neighbourhood $V$ of $p$ such that all trajectories starting in $V$ converge to $p$ without leaving the set $U$. Because $K_{i} \rightarrow\{p\}$, for any neighbourhood $U$ of $p$, there exists $\lambda \in \mathbb{Z}_{>0}$ such that $K_{\ell} \subset U$. If we set $V=K_{\ell+\mu}$, for sufficiently large $\mu \in \mathbb{Z}_{>0}$, then the sets $U$ and $V$ satisfy the conditions for Lyapunov stability and, therefore, the closed-loop system with the piecewise analytic feedback we constructed above is Lyapunov stable.

The verification that there is a well-defined map $E$ so that $\left(\Sigma,\left(\Sigma_{1}, \Sigma_{2}\right),\left\{V_{S}\right\}_{S \in \Sigma_{1}}, E\right)$ is a piecewise analytic feedback for $f$ is the same as in [80] and we conclude this proof by recalling the main idea behind it. Let $q$ be a point in a stratum $S \in \Sigma_{2}$ and such that $q \neq p$. Then $S \subset H_{j}$, for some $j>0$, and we denote by $\gamma$ the integral curve of $Y_{j}$ through $q$. For $\delta>0$ small enough, the compact set $\Gamma=\gamma([0, T])$ intersects finitely many strata $S_{1}, \ldots, S_{r} \subset H_{j}, r \in \mathbb{Z}>0$, and we define $\tilde{S}_{i}=\left\{s \in[0, \delta] \mid \gamma(s) \in \Gamma \cap S_{i}\right\}, i=1, \ldots, r$. The subanalytic sets $\tilde{S}_{i}, i=1, \ldots, r$, form a partition of $[0, \delta]$ and each $\tilde{S}_{i}$ is a finite union of intervals and singletons. Without loss of generality, assume that $q \in S_{1}$; then, there does not exist an interval $[0, \alpha], \alpha \in \mathbb{R}_{>0}$, contained in $\tilde{S}_{1}$ for otherwise $S_{1} \in \Sigma_{1}$, contradicting the hypothesis that $S \in \Sigma_{2}$. Therefore, there exists $k \in\{1, \ldots, r\}$ such that $\gamma(s) \in S_{k}$ for $s \in(0, \alpha), \alpha \in \mathbb{R}_{>0}$, and $S_{k} \in \Sigma_{1}$. Then, in the notation of Section 3.3.1, $E(q)=S_{k}$.

Theorem 3.3 is the core contribution of the present thesis and is an extension to locally controllable systems of Theorem 2.18, by Sussmann [80], for globally controllable systems. To show that the sets $R_{i}$ can be covered by sets of the form $B_{q}$ (in the notation of the proof 
of Theorem 3.3), Sussmann argues as follows: by Sard's theorem, the map $\tau \mapsto \Phi^{\xi}(\tau, p)$ has full rank on an open and dense subset of its domain of definition, for any fixed $k$-tuple $\xi$ of vector fields. Then, given any point $q$ in the reachable (from $p$ ) set of $-f$, there exist $\tau_{1}$ and $\xi_{1}$ such that $\Phi^{\xi_{1}}(\cdot, p)$ has full rank at $\tau_{1}$. If $q_{1}$ is the point $\Phi^{\xi_{1}}\left(\tau_{1}, p\right)$, then, by the assumption of global controllability, there exist $\tau_{2}$ and $\xi_{2}$ such that $\Phi^{\xi_{2}}\left(\tau_{2}, q_{1}\right)=q$, and the overall concatenation of flows from $p$ to $q$, through $q_{1}$, can be shown to have rank equal to the dimension of $M$. Therefore, the construction of the piecewise analytic feedback in [80] relies heavily on the assumption of global controllability that allows any two points - in the argument above, the points $q_{1}$ and $q$-in the state space $M$ to be connected by trajectories of $-f$. When the assumption of local controllability is substituted for that of global controllability, it is not true in anymore that any two points in $M$ can always be connected by trajectories of $-f$ and it is precisely the property of normal reachability that allows the construction of a piecewise analytic feedback to be reinstated.

\subsection{A corollary}

As mentioned in the Introduction, controllability of the unstable eigenvalues of a linear control system is equivalent to the existence of a linear asymptotically stabilising feedback. In other words, it suffices to be able to control the unstable dynamics in order to stabilise a system. Theorem 3.3 can be used to obtain an analogous result for nonlinear control systems and the following corollary formalises this idea.

Let $f: M \times \Omega \rightarrow T M$ be a control system with $\operatorname{dim} M=n$. We first perform some constructions with vector fields following [82]. Let $\mathscr{C}$ be the Lie algebra of vector fields generated by the vector fields $f_{\omega}, \omega \in \Omega$. Let $\mathscr{D} \subset \mathscr{C}$ be the derived algebra which can be shown to be the set of all finite $\mathbb{R}$-linear combinations of vector fields from the set

$$
\left[f_{\omega_{1}},\left[f_{\omega_{2}}, \ldots,\left[f_{\omega_{k-1}}, f_{\omega_{k}}\right]\right]\right], \quad k \geq 2, \omega_{1}, \ldots, \omega_{k} \in \Omega
$$

Let $\mathscr{X}_{0}$ be the family of vector fields

$$
\mathscr{X}_{0}=\left\{\lambda_{1} f_{\omega_{1}}+\cdots+\lambda_{k} f_{\omega_{k}} \mid k \geq 1, \omega_{1}, \ldots, \omega_{k} \in \Omega, \lambda_{1}+\cdots+\lambda_{k}=0\right\} .
$$

Then take $\mathscr{C}_{0}=\mathscr{X}_{0}+\mathscr{D}$; this is a family of vector fields that can be shown to be closed under Lie bracket. Moreover, this family of vector fields is invariant under the vector fields $f_{\omega}, \omega \in \Omega$, in the sense that $\left[f_{\omega}, X\right] \in \mathscr{C}_{0}$ for every $X \in \mathscr{C}_{0}$. Denote by $C_{0}$ the distribution generated by the family of vector fields $\mathscr{C}_{0}$. If $C_{0}$ has constant rank $k$ in a neighbourhood of $p \in M$, then we can find local coordinates $x=\left(x^{1}, \ldots, x^{k}, x^{k+1}, \ldots, x^{n}\right)=\left(y^{1}, y^{2}\right)$ around

$p$ such that $C_{0}=\operatorname{span}\left\{\frac{\partial}{\partial x^{1}}, \ldots, \frac{\partial}{\partial x^{k}}\right\}$ and, because $C_{0}$ is invariant under $f$, we have the local decomposition [82]:

$$
\begin{aligned}
& \dot{y}^{1}=f^{1}\left(y^{1}, y^{2}, \omega\right) \\
& \dot{y}^{2}=f_{0}^{2}\left(y^{2}\right) .
\end{aligned}
$$

3.4 Corollary: If the subsystem $f^{1}$ is locally controllable from $p$ and the vector field $f_{0}^{2}$ has $p$ as a locally asymptotically stable equilibrium, then the control system $f$ is asymptotically stabilisable at $p$.

Obviously, variations of Corollary 3.4 can be obtained by altering the type of stability of the uncontrolled dynamics $f_{0}^{2}$. 


\subsection{Examples}

Consider the question of whether some kind of stabilising feedback exists for the class of control-affine systems described by

$$
\begin{aligned}
& \dot{x}=u, \\
& \dot{y}=Q(x),
\end{aligned}
$$

where $(x, y) \in \mathbb{R}^{m} \times \mathbb{R}^{n}, Q$ is a quadratic form, ${ }^{3}$ and $u \in U \subset \mathbb{R}^{m}$. The control set $U$ is assumed to be compact, convex, and containing a neighbourhood of the origin. The linearisation of (3.6.1) is not controllable, and methods such as the use of Centre Manifold theory $[67$, Ch. 10] or homogeneity arguments $[42,50]$ become impractical as the complexity of $Q$ and the dimension $n$ (of the centre manifold) increase. It has been proven, though, that control systems of the form (3.6.1) are locally controllable from the origin $(0,0) \in \mathbb{R}^{m} \times \mathbb{R}^{n}$ if and only if $Q$ is indefinite [49]. Therefore, if $Q$ is indefinite, Theorem 3.3 implies that there exists a piecewise analytic feedback that asymptotically stabilises (3.6.1) to the origin.

The control-affine system of Example 2.9, that is, $\Sigma: \dot{\mathbf{x}}=X(\mathbf{x})+u Y(\mathbf{x}), \mathbf{x}=(x, y, z) \in$ $\mathbb{R}^{3}, u \in U \subset \mathbb{R}$, where

$$
X(\mathbf{x})=\left[\begin{array}{c}
0 \\
x^{2}(y-x) \\
x^{2}(z-y)
\end{array}\right], \quad Y(\mathbf{x})=\left[\begin{array}{l}
1 \\
0 \\
0
\end{array}\right]
$$

and $U$ is as in the previous example, is locally controllable from the origin, asymptotically controllable to the origin, and it satisfies Coron's condition for stabilisation [26]. However, as is shown in [26], there does not exist a continuous feedback that asymptotically stabilises $\Sigma$ to the origin. It is also remarked in [26] that it is not known whether $\Sigma$ can be stabilised using dynamic feedback. Since $\Sigma$ is locally controllable, we know from Theorem 3.3 that it can be locally asymptotically stabilised to the origin using a piecewise analytic feedback.

Our last example is meant to illustrate the behaviour of control systems when the rank condition of Corollary 3.4 does not hold. It is an instance of how the notion of singularities, if properly understood, will clarify further the relationship between controllability and stabilisability for nonlinear control systems, and indeed the properties of these systems in general. It is worth noting the simplicity of the example.

The control system

$$
\dot{x}=(1-u) x,
$$

where $x, u \in \mathbb{R}$, becomes asymptotically stable at $x=0$ for any choice of a constant control with value greater than one. At the same time, this system is clearly not locally controllable from $x=0$.

\footnotetext{
${ }^{3}$ See Problem 10.4 in $[17$, p. 315] and the references therein for the long history of vector-valued quadratic forms and their relevance to control theory.
} 


\section{Chapter 4}

\section{A converse Lyapunov-type theorem for locally controllable systems}

\subsection{Introduction}

4.1.1. Lyapunov stability and converse Lyapunov theorems. In his 1892 dissertation, Lyapunov made a fundamental contribution to the theory of stability for dynamical systems by introducing a powerful technique which allows the stability analysis of a dynamical system without explicit knowledge of the trajectories of the system [61, 68]. The central idea in Lyapunov's work is to define a so-called Lyapunov function which can be viewed as a generalised energy and prove that, if such a function exists and is decreasing along the trajectories of a dynamical system, then the dynamical system has to be stable, mimicking thus the process of energy decay for dissipative physical systems. Mathematicians, naturally, followed up Lyapunov's accomplishment by asking whether Lyapunov's stability and instability theorems admit converses; that is, in the case of the stability theorems, whether Lyapunov functions exist for stable dynamical systems. The affirmative answer to this question was given by several mathematicians [12], [44, Ch.VI] at varying levels of generality. In the case of dynamical systems defined by differential equations, the most general results are due to Kurzweil [57], Massera [64], and Zubov [88]. Kurzweil and Massera independently proved that it suffices for the right-hand side of a differential equation to be a continuous function for a $C^{\infty}$ Lyapunov function to exist. They also showed that if the differential equation is periodic or time-independent, then there exists a Lyapunov function having the same property. Zubov, on the other hand, introduced a general method for stability analysis that he applied both to dynamical systems and differential equations. We comment in more detail on Zubov's work below, since we employ some of the core ideas of his approach in the present chapter. It is worth noting that converse Lyapunov theorems not only answer a mathematically interesting question, but also lead to a better understanding of the stability properties of dynamical systems. The paper [63] is a representative example where it is shown that the assumptions in Lyapunov's second theorem imply equiasymptotic stability ${ }^{1}$, a type of stability which is stronger than asymptotic stability ${ }^{2}$, and they

\footnotetext{
${ }^{1}$ That is, asymptotic stability with the additional property that convergence to the equilibrium is uniform with respect to the initial condition of the trajectories.

${ }^{2}$ In the non-autonomous case, otherwise the two notions are equivalent.
} 
have to be weakened for a converse theorem to hold. As far as the literature on classical stability theory is concerned, in addition to the monographs [12] and [44], the paper [64] provides a useful roadmap to earlier results due to Barbašin, Krasovskiı̌, Četaev, Malkin, Persidskiı, and Yoshizawa. Entry points to the literature on extensions and generalisations of Lyapunov's ideas such as piecewise smooth Lyapunov functions, prolongations, or stability theorems that involve second or higher derivates of Lyapunov functions can be found in [12]. Numerous sources of historical information on stability theory are available; the recent publication [62] on Lyapunov stability has the additional quality of clarifying misattributions and misnomers in the stability literature.

4.1.2. Zubov's method. Zubov's work on stability stands out for a multitude of reasons. ${ }^{3}$ To motivate the rest of the chapter, we explain in the present section its relevance to our framework and why it provides a natural approach to the problem at hand, i.e., the proof of the existence of a Lyapunov function for small-time locally controllable systems.

As we mentioned above, Zubov introduced a method that applies not only to differential equations but also to dynamical systems on metric spaces [88, Ch.I]. This aspect of the method is important to us for the following reason. To prove the existence of a Lyapunov function for real analytic, small-time locally controllable systems, we first show that there exists a piecewise analytic feedback that stabilises the control system in finite time. The existence of the feedback is proven using a stratification theorem for subanalytic sets [80] and, therefore, a closed-form expression - that is, a formula - describing the feedback is not available. However, the resulting closed-loop system induces a dynamical system on the state space of the control system and, hence, we can still apply Zubov's method.

The second aspect of Zubov's method that is important to us is its versatility that allows for different definitions of a dynamical system. Specifically, in our proof of stabilisability (Theorem 4.2) the trajectories of the dynamical system induced on the state space by the closed-loop are not continuous with respect to initial conditions and they converge in finite time to the equilibrium from which the control system is small-time locally controllable. Because of these characteristics, the dynamical system and its stability properties do not satisfy some of the assumptions classically made $[11,12,44,88]$ to prove converse Lyapunov theorems. Of course, in the modern theory of dynamical systems [65] the classical assumptions have been considerably relaxed. However, we aim for a balance between the generality of the assumptions we make and the strength of the conclusions we draw. To wit, although it is possible, for example, to prove converse Lyapunov theorems for dynamical systems that are discontinuous with respect to both the time and state variables and that satisfy conditions weaker than the semi-group property (see for example [65, Theorem 3.6.1]), we wish to exploit to the largest possible extent the known structure of the control system and, consequently, of the dynamical system at hand, to sharpen our result.

One of the main contributions of Zubov [57, p. 21] is the characterisation of the domain of attraction of an asymptotically stable equilibrium as the sub-level set of a suitably defined Lyapunov function. Although not necessary from a strictly logical point of view for what we want to accomplish here, this characterisation provides an interesting connection between the reachable set of the control systems we consider and the domain of definition of a Lyapunov function. This connection has not been fully explored yet.

\footnotetext{
${ }^{3}$ Lefschetz describes [88] as a "first rate book" and its last chapter as "the most striking part".
} 
4.1.3. Control theory. From a control-theoretic point of view, Theorem 4.3 establishes a connection between local controllability and the existence of a Lyapunov function. It is perhaps interesting to view this connection as another necessary condition for local controllability. The idea of using Zubov's method in control theory is not new, of course. It is well known that control theory has been heavily influenced by the theory of Lyapunov stability, especially through the generalisation of Lyapunov functions to control-Lyapunov functions [78, Ch.5] and through foundational contributions - analogous to the ones in the classical theory of stability - such as the first converse theorems for control-Lyapunov functions [5]. In fact, the Lyapunov approach constitutes the main paradigm in the study of the stabilisation problem, one of the fundamental problems in control theory. In the opposite direction, significant impetus for the theoretical developments has been provided by the successful application of control-Lyapunov functions to control engineering problems and more specifically to the design of robust feedback controllers [34, 56]. The literature on control-Lyapunov functions and their application to control theoretic problems is now enormous; a review of many significant results can be found in [7]. As far as the application of Zubov's method to control theory is concerned, the main guiding principle that led to important generalisations and progress in understanding the relationship between contol-Lyapunov functions and stabilisation has been to obtain a control-Lyapunov function as a viscosity solution of a PDE of Hamilton-Jacobi type that generalises Zubov's equation $[8,19,20,34,43]$. In this context, small-time local controllability becomes especially relevant since it is known $[33,81]$ that it implies the continuity of the minimum-time function. In fact, one has the following theorem. The terms used in the statement of the theorem should be self-explanatory; the definitions can be found in [21] where a synthesising overview of the contributions to control theory of the school of non-smooth analysis can also be found.

4.1 Theorem: A small-time locally controllable system is globally asymptotically controllable in finite time if and only if there exists a Lyapunov pair $(V, W)^{4}$ with $V$ continuous and $W \equiv 1$.

Several authors $[7,8,21]$ define small-time local controllability to be controllability to a point. Being a definition, it could be anything meaningful, however the majority of the literature defines small-time local controllability to be controllabiltiy from a point. The equivalence of the two definitions stems from a nontrivial theorem originally conjectured by Sussmann and proven by Grasse [40], the point being that the same term is used for two logically equivalent but different properties. What is obvious, of course, is that small-time local controllability from a point is equivalent to small-time local controllability to that point for the time-reversed system.

Theorem 4.1 bears some similarity to our Theorem 4.3. One way our result is different from Theorem 4.1, and other similar results, is that we prove the existence of a Lyapunov function for a dynamical system that corresponds to (the closed-loop system of) a control system and we do not deal with open loop controls. Our Lyapunov function is not necessarily continuous, however it has a derivative in the usual ("strong") sense along the trajectories of the closed-loop system. Moreover, we believe that a distinctive feature of our proof is its simplicity: the construction of the Lyapunov function and the proof the accompanying

\footnotetext{
${ }^{4}$ The function $V$ is the control-Lyapunov function and the function $W$ is the positive definite function that bounds the negative of a generalised derivative (sub-differential) of $V$ with respect to the control system.
} 
properties use only elementary arguments.

Although our focus is exclusively on small-time locally controllable systems, we would like to end the present section with a few landmarks in the development of the (control) Lyapunov theory for asymptotically controllable systems and, more specifically, with results on converse Lyapunov-type theorems. To keep the size of this already long literature review reasonable, we will not digress into the related, but different, topic of how Lyapunov functions, smooth or not, can be used to construct feedback controls, however we will mention such results if they seem necessary to make the picture coherent.

One of the earliest general results on asymptotic controllability is a Lyapunov characterisation by Sontag [77] which provided the basis for proving in [22] that a sampled-data feedback exists for asymptotically controllable systems. The feedback in [22] is obtained by solving a sequence of optimisation problems, each one of them being initialised by sampling the state of the system. As the authors point out, the feedback so constructed is not robust with respect to state measurement errors, however, subsequent work [59,69] improved on this aspect of the result. A different approach to the problem of showing the existence of stabilising feedbacks for asymptotically controllable systems is taken in [3] were it is shown that there exist families of vector fields that can be patched together to construct an asymptotically stable closed-loop. Results on the robustness properties of these patchy feedbacks are presented in [4]. Building on [3], the authors of [39] use the existence of asymptotically stabilising patchy feedbacks to prove the existence of piecewise smooth patchy controlLyapunov functions for asymptotically controllable systems. In the present chapter, we prove the existence of a stabilising feedback and use this fact to prove the existence of a Lyapunov function and, in this sense, the philosophy of our approach parallels that of [39]. As mentioned above, the paper [22] follows the opposite direction by proving the existence of a stabilising feedback, given the existence of a Lyapunov function. Other papers that contain recent results of a general nature on the existence of stabilising feedbacks and on Lyapunov-like characterisations of control-theoretic properties are [54, 66, 70, 71, 84].

\subsection{Stabilisation of locally controllable systems in finite time}

The first step in proving the existence of a Lyapunov function for locally controllable systems is to show the stabilisability of the latter. Although it was shown in Chapter 3 that STLC systems can, indeed, be asymptotically stabilised, in this section we show that a slight modification of the proof of Theorem 3.3 yields stabilisation of STLC systems in finite time. The rationale behind this approach is twofold: first, it gives a different result which is interesting in itself; second, showing that the trajectories of the closed-loop system converge to the equilibrium in finite time greatly simplifies the arguments related to the existence and the properties of the Lyapunov functions. It is perhaps interesting to observe that stabilisation in finite time comes at the expense of a closed-loop system which may not be Lyapunov stable. Whether the two properties can be reconciled requires further investigation.

As mentioned already, the proof of the Theorem 4.2 is similar to that of Theorem 3.3, but presenting only the necessary modifications with constant references to the proof of Theorem 3.3 would make the exposition too convoluted.

4.2 Theorem: If a control system $f: M \times \Omega \rightarrow T M$ is locally controllable from an equilib- 
rium $p \in M$ and admits a uniform bound, and if $\Omega$ is compact and $\{f(p, \omega) \mid \omega \in \Omega\}$ is convex for every $p \in M$, then there exists a piecewise analytic feedback that locally stabilises $f$ at $p$ in finite time.

Proof: If $\xi=\left(X_{1}, \ldots, X_{k}\right)$ is a finite sequence of vector fields, $|\xi|$ denotes the number of elements in $\xi$, i.e. $|\xi|=k$, and if $\tau=\left(t_{1}, \ldots, t_{k}\right)$ is a $k$-tuple of real numbers, $|\tau|$ is equal to the sum of the components of $\tau$, i.e., $|\tau|=t_{1}+\cdots+t_{k}$. Given such a $\tau \in \mathbb{R}^{k}$, we denote the cubical neighbourhood of $\tau$ of side $2 \varepsilon$ by $C_{\varepsilon}^{k}(\tau)$; in other words,

$$
C_{\varepsilon}^{k}(\tau)=\left\{\left(s_{1}, \ldots, s_{k}\right) \in \mathbb{R}^{k}|| t_{i}-s_{i} \mid \leq \varepsilon, i \in\{1, \ldots, k\}\right\} .
$$

The non-negative orthant of $\mathbb{R}^{k}$ will be denoted by $\mathbb{R}_{\geq 0}^{k}$ and it is, by definition, the set $\left\{\tau=\left(t_{1}, \ldots, t_{k}\right) \in \mathbb{R}^{k} \mid t_{i} \geq 0, i \in\{1, \ldots, k\}\right\}$. Also, for the ease of notation, if

$$
\Phi^{f}: \mathbb{R} \times M \supset U \ni(t, p) \mapsto \Phi^{f}(t, p) \in M
$$

is the flow (defined on some open subset $U$ ) of a vector field $f$ on a manifold $M$, we write $\Phi_{p}^{f}$ for the map $t \mapsto \Phi^{f}(t, p)$. For a collection of vector fields $\xi$ and a $k$-tuple $\tau$ as above, $\Phi_{\tau}^{\xi}$ stands for the composition $\Phi_{t_{1}}^{X_{1}} \circ \cdots \circ \Phi_{t_{k}}^{X_{k}}$ and, similarly to the case of one vector field just described, $\Phi_{p}^{\xi}$ is the map $\tau \mapsto \Phi^{\xi}(\tau, p)$.

Since the control system $f$ is locally controllable from $p$, the time-reversed system $-f$ is also locally controllable from $p$, by Theorem 3.2. By the same theorem, if $q$ is a point in the reachable set $R_{-f}(p,[0, T])$, then $q$ is normally reachable from $p$ via $-f$ in time less than $T+\alpha$, where $\alpha$ is an arbitrarily small positive real number. One way to see this is to observe that, since $p$ is an equilibrium for $f, R_{-f}(p,[0, T]) \subset R_{-f}(p,[0, T+\alpha))$. Theorem 3.2 can then be applied directly to the set $R_{-f}(p,[0, T+\alpha))$. Normal reachability of $q$ from $p$ via $-f$ in time less than $T+\alpha$ means precisely that we can find a finite sequence of vector fields $\xi_{q}=\left(X_{1}, \ldots, X_{\left|\xi_{q}\right|}\right)$ and $\sigma_{q}=\left(s_{1}, \ldots, s_{\left|\xi_{q}\right|}\right) \in \mathbb{R}_{\geq 0}^{\left|\xi_{q}\right|}$ such that $\left|\sigma_{q}\right|<T+\alpha$, each $X_{i}$, $i \in\left\{1, \ldots,\left|\xi_{q}\right|\right\}$, is of the form $-f_{\omega}$, and $\Phi^{\xi_{q}}\left(\sigma_{q}, p\right) \triangleq \Phi_{s_{1}}^{X_{1}} \circ \cdots \circ \Phi_{s_{\mid \xi q} \mid}^{X_{\left|\xi_{q}\right|}}(p)=q$, with the map $\Phi_{p}^{\xi_{q}}$ having rank equal to the dimension of $M$ at $\sigma_{q}$. Since the rank of the map $\Phi_{p}^{\xi_{q}}$ is $\operatorname{dim} M$ at $\sigma_{q}$, there exists $\varepsilon_{q}>0$ such that the map $\Phi_{p}^{\xi_{q}}$ is defined on $C_{\varepsilon_{q}}^{\left|\xi_{q}\right|}\left(\sigma_{q}\right) \cap \mathbb{R}_{\geq 0}^{\left|\xi_{q}\right|}$ and the set $\Phi_{p}^{\xi_{q}}\left(C_{\varepsilon_{q}}^{\left|\xi_{q}\right|}\left(\sigma_{q}\right) \cap \mathbb{R}_{\geq 0}^{\left|\xi_{q}\right|}\right)$ contains a neighbourhood of $q$.

For every $\tau \in C_{\varepsilon_{q}}^{\left|\xi_{q}\right|}\left(\sigma_{q}\right) \cap \mathbb{R}_{\geq 0}^{\left|\xi_{q}\right|}$, we can define a curve

$$
\eta_{\tau}:[0,|\tau|] \ni t \mapsto \eta_{\tau}(t) \in \mathbb{R}^{\left|\xi_{q}\right|}
$$

such that $t \mapsto\left(\Phi_{p}^{\xi_{q}} \circ \eta_{\tau}\right)(t)$ is the concatenation of trajectories of the control system $-f$ that connects $p$ and $\Phi_{p}^{\xi_{q}}(\tau)$ (see Figure 3.1). Let $A_{q}$ denote the set of points in $\mathbb{R}_{\geq 0}^{\left|\xi_{q}\right|}$ which are of the form $\eta_{\tau}(t), \tau \in C_{\varepsilon_{q}}^{\left|\xi_{q}\right|}\left(\sigma_{q}\right) \cap \mathbb{R}_{\geq 0}^{\left|\xi_{q}\right|}, t \in[0,|\tau|]$. The set $A_{q}$ can be written as a finite union of compact semianalytic sets: $A_{q}=A_{q}^{1} \cup \cdots \cup A_{q}^{\left|\xi_{q}\right|}$. The geometric meaning of the $A_{q}^{i}$ is shown in Figure 3.2; they are sets of increasing dimension and every $A_{q}^{i}$ is a rectangular "neighbourhood" of the $i$ th segment of the curve $\eta_{\tau}$. The explicit description of the sets $A_{q}^{i}$ in terms of inequalities - inequalities that stem form the fact that $\tau \in C_{\varepsilon_{q}}^{\left|\xi_{q}\right|}\left(\sigma_{q}\right) \cap \mathbb{R}_{\geq 0}^{\left|\xi_{q}\right|}$ and $t \in[0,|\tau|]$ - is as follows [80, p. 45]: the set $A_{q}^{i}$ consists of those points $\left(t_{1}, \ldots, t_{\left|\xi_{q}\right|}\right)$ that satisfy 
(i) $t_{j}=0$, for $j \leq\left|\xi_{q}\right|-i$,

(ii) $0 \leq t_{j} \leq b_{j}$, for $j=\left|\xi_{q}\right|+1-i$, and

(iii) $a_{j} \leq t_{j} \leq b_{j}$, for $j>\left|\xi_{q}\right|+1-i$,

where $a_{q}^{i}=\max \left(\sigma_{q, i}-\varepsilon_{q}, 0\right), b_{q}^{i}=\sigma_{q, i}+\varepsilon_{q}$, and $\sigma_{q, i}$ denotes the $i$ th component of $\sigma_{q}$. Since the sets $A_{q}, A_{q}^{i}$ are compact semianalytic, their images $B_{q}=\Phi_{p}^{\left|\xi_{q}\right|}\left(A_{q}\right), B_{q}^{i}=\Phi_{p}^{\left|\xi_{q}\right|}\left(A_{q}^{i}\right)$ are compact subanalytic sets and the set $B_{q}$ contains a neighbourhood of the point $q$.

Consider now a strictly increasing sequence $\left(T_{i}\right)$ of positive real numbers and the corresponding increasing sequence of compact sets $K_{i} \triangleq R_{-f}\left(p,\left[0, T_{i}\right]\right)$; compactness follows from the assumptions of our theorem [60, p. 242]. Each point $q$ in $R_{i} \triangleq K_{i+1} \backslash \operatorname{int} K_{i}$ is contained in a set $B_{q}$ of the form described above and, therefore, by successively covering each compact set $R_{i}$ with finitely many sets $B_{q}$ we obtain a countable covering $\left(B_{q_{m}}\right)$ of the set $\mathscr{R} \triangleq R_{-f}(p,[0, \infty))$. Following Sussmann's construction [80], we use the indices $i$, $j$, and $m$ uniquely defined by the relation

$$
j=i+\left|\xi_{q_{1}}\right|+\cdots+\left|\xi_{q_{m-1}}\right|
$$

with $1 \leq i \leq\left|\xi_{q_{m}}\right|$, to define the sequence of sets $D_{j}=B_{q_{m}}^{i}$, with $D_{0}=\{p\}$. Then $E_{j}=D_{0} \cup \cdots \cup D_{j}$ is an increasing sequence of compact subanalytic sets and we set $H_{j}=$ $E_{j} \backslash E_{j-1}$, with $E_{-1}=\emptyset$. The collection $\left\{H_{j}\right\}_{j \geq 0}$ of sets is, then, a locally finite partition of $\mathscr{R}$ into relatively compact subanalytic sets. If we assign the vector field $Y_{j}=-X_{\left|\xi_{q_{m}}\right|+1-i}$ to each set $H_{j}, j \geq 1$, and the vector field $Y_{0}=0$ to the set $H_{0} \triangleq\{p\}$, we can apply Theorem 3.1 to the family $\mathscr{H}=\left\{H_{j}\right\}_{j \geq 1}$ of subanalytic subsets, with $F\left(H_{j}\right)=\left\{Y_{j}\right\}$ (see the notation in the theorem), to obtain a stratification $\Sigma$ of $\mathscr{R}$, compatible with the family $\mathscr{H}$. The stratification $\Sigma$ is partitioned into $\Sigma_{1}$ and $\Sigma_{2}$ (see Section 3.3.1) in the following way: given that every stratum $S \in \Sigma$ is a subset of an $H_{j}$ for some $j$, if $Y_{j}$ is everywhere tangent to $S$ then $S \in \Sigma_{1}$, otherwise $S \in \Sigma_{2}$. If we set $V_{S}=Y_{j}$, for all $S \in \Sigma_{1}$, then $\mathscr{F}=\left(\Sigma,\left(\Sigma_{1}, \Sigma_{2}\right),\left\{V_{S}\right\}_{S \in \Sigma_{1}}, E\right)$ is a piecewise analytic feedback for $f$ that steers every point of $\mathscr{R}$ to $p$ in finite time [80, p. 46-48].

\subsection{Proof of the main result}

In the previous section we showed that STLC systems can be locally stabilised in finite time using a piecewise analytic feedback. In the present section we explain how the flow of the resulting closed-loop system defines on the state space a dynamical system which provides a convenient formalism for the proof of our converse Lyapunov theorem. Let us begin by recalling the definition of an action.

A (left) action of a monoid $G$ on a metric space $X$ is a map $\phi: G \times X \rightarrow X$ that satisfies the following properties:

(i) $\phi\left(1_{G}, x\right)=x, \forall x \in X$;

(ii) $\phi\left(g_{2} g_{1}, x\right)=\phi\left(g_{2}, \phi\left(g_{1}, x\right)\right), \forall g_{1}, g_{2} \in G, \forall x \in X$,

where $1_{G}$ is the identity in $G$. Because the dynamical system associated with the piecewise analytic feedback of the previous section is defined only for positive times - we follow the 
common convention according to which we label as "time" the variable that takes its values in $G$-and it has trajectories that do not depend continuously on their initial conditions, we are lead to the following definition. A dynamical system on a metric space $X$ is an action of a monoid $G$ on $X$. It is perhaps worth noting at this point that stronger definitions of a dynamical system satisfy a fortiori the definition just given and, hence, we have not tailored our definition to our convenience. In other words, if, for example, negative times are allowed and $G$ has the structure of a group, then $G$ is also a monoid. If continuity with respect to initial conditions holds then the map $\phi$ will satisfy additional continuity properties. In fact, our $\phi$ is continuous with respect to time. ${ }^{5}$

The following definition relies on the fact that, to each initial condition $q \in \mathscr{R}$, there corresponds a unique trajectory of the piecewise analytic feedback we constructed in the previous section. Such a trajectory is a concatenation of integral curves of analytic vector fields of the form $f(x, \omega)$, with $\omega \in \Omega$ fixed. If $d$ denotes a metric on $M$ that generates the topology of $M$ (see the beginning of Section 3.2 for the defining properties of a manifold that imply its metrisability), the dynamical system that corresponds to the flow of a piecewise analytic feedback is defined as follows.

Let $f: M \times \Omega \rightarrow T M$ and $p \in M$ be as in Theorem 4.2, and $\mathscr{F}$ a piecewise analytic feedback for $f$. The dynamical system $\widetilde{\mathscr{F}}$ is the action $\phi$ of $G=\left(\mathbb{R}_{\geq 0},+\right)$ on $X=(\mathscr{R}, d)$, where $\mathscr{R}=R_{-f}(p,[0, \infty))$, defined as follows: given $t \in G$ and $q \in \mathscr{R}, \phi(t, q)$ is the point in $\mathscr{R}$ that is reached by following for time $t$ the trajectory of $\mathscr{F}$ that starts at $q$ at time 0 . Moreover, for all $t \in G$, we set $\phi(t, p)=p$.

In what follows, we write interchangeably $\phi(t, q)$ and $t q$ for the value of $\phi$ at $(t, q) \in$ $G \times \mathscr{R}$. In the latter notation, associativity of the action is expressed as $\left(t_{2}+t_{1}\right) q=t_{2}\left(t_{1} q\right)$. Given $q \in \mathscr{R}$, we define the trajectory through $\boldsymbol{q}$ of $\widetilde{\mathscr{F}}$ to be the map $\gamma_{q}: G \ni t \mapsto$ $\phi(t, q) \in \mathscr{R}$. Being a concatenation of integral curves of analytic vector fields, $\gamma_{q}$ is a continuous map. We have now established the necessary framework to prove the following.

4.3 Theorem: Let $f: M \times \Omega \rightarrow T M$ be a control system, $p \in M$ an equilibrium, and $\mathscr{F}$ a stabilising piecewise analytic feedback as in Theorem 4.2. There exists a positive-definite function $V$ defined on $\mathscr{R}$ such that, for all $q \in \mathscr{R}$, the derivative of $V$ along $\gamma_{q}$ negative definite.

Proof: Define on $\mathscr{R}=R_{-f}(p,[0, \infty))$ the function

$$
V: \mathscr{R} \ni q \mapsto 1-e^{-\int_{0}^{\infty} d(t q, p) \mathrm{d} t} \in \mathbb{R} .
$$

Every point $q \in \mathscr{R}$ converges to $p$ in some finite time $T_{q}>0$ and, therefore,

$$
\int_{0}^{\infty} d(t q, p) \mathrm{d} t=\int_{0}^{T_{q}} d(t q, p) \mathrm{d} t<\infty
$$

which shows that $V$ is well-defined. Since, for any $q \in \mathscr{R} \backslash\{p\}$, there exist $\eta>0$ and

\footnotetext{
${ }^{5}$ In the manifold topology.
} 
$T_{\eta} \in\left(0, T_{q}\right)$ such that $d(t q, p)>\eta$ for $t \in\left[0, T_{\eta}\right]$, it follows that

$$
\begin{aligned}
& \int_{0}^{T_{\eta}} d(t q, p) \mathrm{d} t>\int_{0}^{T_{\eta}} \eta \mathrm{d} t=\eta T_{\eta} \\
\Rightarrow & -e^{-\int_{0}^{T_{\eta}} d(t q, p) \mathrm{d} t}>-e^{-\eta T_{\eta}} \\
\Rightarrow & 1-e^{-\int_{0}^{T_{\eta}} d(t q, p) \mathrm{d} t} e^{-\int_{T_{\eta}}^{T_{q}} d(t q, p) \mathrm{d} t}>1-e^{-\eta T_{\eta}} e^{-\int_{T_{\eta}}^{T_{q}} d(t q, p) \mathrm{d} t} \\
\Rightarrow & V(q)>1-e^{-\eta T_{\eta}} e^{-\int_{T_{\eta}}^{T_{q}} d(t q, p) \mathrm{d} t} \geq 1-e^{-\eta T_{\eta}}>0 .
\end{aligned}
$$

To go from the third to the last line we used Eq. (4.3.2), whereas for the last two inequalities we used the facts that $d(t q, p) \geq 0, t \in\left[T_{\eta}, T_{q}\right]$, and $\eta T_{\eta}>0$, respectively. Moreover,

$$
V(p)=1-e^{-\int_{0}^{\infty} d(t p, p) \mathrm{d} t}=1-e^{0}=0 .
$$

We have thus shown that $V$ is positive definite. We complete the proof by showing that the derivative of $V$ along the trajectories of $\widetilde{\mathscr{F}}$ is negative definite. From the definition of the function $V$, we have for $q \in \mathscr{R} \backslash\{p\}$ and $t \in[0, \infty)$

$$
\begin{aligned}
1-V(q) & =e^{-\int_{0}^{\infty} d(s q, p) \mathrm{d} s} \\
& =e^{-\int_{0}^{t} d(s q, p) \mathrm{d} s} e^{-\int_{t}^{\infty} d(s q, p) \mathrm{d} s} \\
\Rightarrow[1-V(q)] e^{\int_{0}^{t} d(s q, p) \mathrm{d} s} & =e^{-\int_{t}^{\infty} d(s q, p) \mathrm{d} s} \\
& =e^{-\int_{0}^{\infty} d((u+t) q, p) \mathrm{d} u} \\
& =e^{-\int_{0}^{\infty} d(u(t q), p) \mathrm{d} u} \\
& =1-V(t q) .
\end{aligned}
$$

A metric is uniformly continuous with respect to the metric structure it defines and, as explained above, the action $\phi(t, q)=t q$ is continuous with respect to $t$. Therefore, $V(t q)$ is differentiable with respect to $t$ and we have

$$
\frac{\mathrm{d} V(t q)}{\mathrm{d} t}=-[1-V(q)] d(t q, p) e^{\int_{0}^{t} d(s q, p) \mathrm{d} s} .
$$

Setting $t=0$ gives

$$
\left.\frac{\mathrm{d} V(t q)}{\mathrm{d} t}\right|_{t=0}=-[1-V(q)] d(q, p)
$$

and both factors on the right-hand side are positive for $q \neq p$. The derivative at any other time $t_{0}$ along the trajectory $\gamma_{q}$ can be computed by a change of variables and is easily seen to be

$$
\left.\frac{\mathrm{d} V(t q)}{\mathrm{d} t}\right|_{t=t_{0}}=\left.\frac{\mathrm{d} V(s \tilde{q})}{\mathrm{d} s}\right|_{s=0},
$$

where $\tilde{q}=t_{0} q$ and the last quantity is negative from the previous calculation. 
One observation that could potentially be interesting, as far as the relation between the function $V$ and the structure of the reachable sets $R_{-f}(p, T)$ is concerned, is the following. If $\mathscr{F}$ is a feedback and $V$ is a function as in Theorem 4.3, then a simple calculation shows that the points $q$ that are steered to $p$ by $\mathscr{F}$ in time exactly $T>0$ are characterised by the relation

$$
V(q)=1-e^{-\int_{0}^{T} d(t q, p) \mathrm{d} t}
$$

where $T$ is the smallest positive real number such that

$$
\left.\frac{\mathrm{d} V(t q)}{\mathrm{d} t}\right|_{t=T}=0
$$

Therefore, the level sets of $V$ correspond to the reachable sets $R_{-f}(p, T)$.

\subsection{Examples}

We begin by revisiting the control-affine system $\Sigma_{1}: \dot{\mathbf{x}}=X(\mathbf{x})+u Y(\mathbf{x}), \mathbf{x}=(x, y, z) \in$ $\mathbb{R}^{3}, u \in U \subset \mathbb{R}$, of Example 2.9, in the context of the present chapter. Recall that

$$
X(\mathbf{x})=\left[\begin{array}{c}
0 \\
x^{2}(y-x) \\
x^{2}(z-y)
\end{array}\right], \quad Y(\mathbf{x})=\left[\begin{array}{l}
1 \\
0 \\
0
\end{array}\right],
$$

and $U$ is assumed to be compact, convex, and containing a neighbourhood of the origin. The control system $\Sigma_{1}$ satisfies both Brockett's [15, p. 181],[87] and Coron's [26] necessary conditions for continuous stabilisability, however, as explained in [26], there does not exist an asymptotically stabilising $u \in C^{0}\left(\mathbb{R}^{3}\right)$ for $\Sigma_{1}$. From Artstein's theorem [5], it follows that a smooth control-Lyapunov function for $\Sigma_{1}$ does not exist as well. However, because $\Sigma_{1}$ is asymptotically controllable to the origin, Sontag's characterisation [77] implies the existence of a continuous control-Lyapunov function. In connection with our result, system $\Sigma_{1}$ can be shown to be STLC from $0 \in \mathbb{R}^{3}$ and, therefore, there exists a function $V$ as in Theorem 4.3. That is, a positive definite $V$ with negative derivative along closed-loop trajectories of $\Sigma_{1}$. It would be interesting to know whether Theorem 4.3 can be improved to yield a continuous $V$.

Our second example is the control-affine system $\Sigma_{2}: \dot{\mathbf{x}}=X(\mathbf{x})+u Y(\mathbf{x})$ of Example 2.15 , where $\mathbf{x}=(x, y) \in \mathbb{R}^{2}, u \in U \subset \mathbb{R}$, with $U$ as in the previous example, and

$$
X(\mathbf{x})=\left[\begin{array}{c}
0 \\
y-x^{3}
\end{array}\right] \quad, \quad Y(\mathbf{x})=\left[\begin{array}{l}
1 \\
0
\end{array}\right] .
$$

Theorem 4.3 can be applied to $\Sigma_{2}$ since the latter is STLC from $0 \in \mathbb{R}^{2}$. However we can draw stronger conclusions in terms of the regularity of the Lyapunov function: as shown in [53], $\Sigma_{2}$ can be asymptotically stabilised using a Hölder-continuous feedback and, by Artstein's theorem, there exists a smooth control-Lyapunov function for $\Sigma_{2}$. Our interest in this example stems from the fact that any map $f: \mathbb{R}^{m} \rightarrow \mathbb{R}^{n}$ with subanalytic graph is locally Hölder-continuous if it is continuous [55, p. 183], and hence a plausible conjecture is that, under additional hypotheses that, for example, must be violated by $\Sigma_{1}$, Theorem 3.3 could yield a Hölder-continuous feedback. 
Lastly, to demonstrate the numerical approximation of the function $V$ of Theorem 4.3, we consider an elementary optimal control problem. Specifically, the problem of driving to the origin in minimum time and with zero terminal speed a point mass that is restricted to move on the real line, starting from any initial condition. The advantage of considering this simple problem is that the optimal feedback can be easily constructed explicitly and is piecewise constant. Therefore, the construction contained in the proof of Theorem 4.3 can be readily applied to yield a Lyapunov function $V$. As is well known, the solution to this optimal control problem is a bang-bang control and the numerical approximation suggests that the function $V$ is piecewise differentiable with the loss of differentiability taking place along the switching locus of the optimal feedback control. The same procedure can, in principle, be applied to any control system for which a feedback $\mathscr{F}$, as in Theorem 4.2, can be constructed, however, in the case of nonlinear control systems, the complexity of constructing $\mathscr{F}$ seems prohibitive at the moment.

A point of unit mass moving without friction on a horizontal track under the influence of a force $u$ whose magnitude can vary from -1 to 1 can be modelled by the control system

$$
\begin{aligned}
& \dot{x}=y, \\
& \dot{y}=u,
\end{aligned}
$$

where $x, y \in \mathbb{R}$, and $u \in[-1,1]$. If we define the function

$$
y=W(x)= \begin{cases}-\sqrt{2 x}, & x \geq 0, \\ \sqrt{-2 x}, & x<0,\end{cases}
$$

and call $\Gamma_{-}$and $\Gamma_{+}$the branches that correspond to $x<0$ and $x \geq 0$, respectively, then the feedback $\Psi$ that steers the point mass from any intial condition to the origin in minimum time and with zero terminal speed is as follows $[60, \mathrm{p} .4]$

$$
\Psi(x, y)= \begin{cases}-1, & (y>W(x)) \vee\left((x, y) \in \Gamma_{-} \backslash\{(0,0)\}\right) \\ 0, & (x, y)=(0,0) \\ 1, & (y<W(x)) \vee\left((x, y) \in \Gamma_{+} \backslash\{(0,0)\}\right)\end{cases}
$$

Given the feedback $\Psi$ and Equation (4.3.1), the function $V$ we are after can be written as

$$
V(x, y)= \begin{cases}V_{u}, & (y>W(x)) \vee\left((x, y) \in \Gamma_{-} \backslash\{(0,0)\}\right) \\ 0, & (x, y)=(0,0) \\ V_{\ell}, & (y<W(x)) \vee\left((x, y) \in \Gamma_{+} \backslash\{(0,0)\}\right)\end{cases}
$$

where

$$
\begin{aligned}
V_{u}=1-\exp \left(-\int_{0}^{t_{s}}\right. & \sqrt{\left(x+t y-t^{2} / 2\right)^{2}+(y-t)^{2}} \mathrm{~d} t- \\
& \left.\int_{t_{s}}^{\infty} \sqrt{\left[t^{2} / 2+t\left(y_{s}-t_{s}\right)-t_{s} y_{s}+t_{s}^{2} / 2+x_{s}\right]^{2}+\left(t-t_{s}+y_{s}\right)^{2}} \mathrm{~d} t\right)
\end{aligned}
$$


and

$$
\begin{aligned}
V_{u}=1-\exp (- & \int_{0}^{t_{s}} \sqrt{\left(x+t y+t^{2} / 2\right)^{2}+(y+t)^{2}} \mathrm{~d} t- \\
& \left.\int_{t_{s}}^{\infty} \sqrt{\left[-t^{2} / 2+t\left(y_{s}+t_{s}\right)-t_{s} y_{s}-t_{s}^{2} / 2+x_{s}\right]^{2}+\left(-t+t_{s}+y_{s}\right)^{2}} \mathrm{~d} t\right) .
\end{aligned}
$$

The integration limit $t_{s}$ is the first time when the trajectory of the closed-loop system

$$
\begin{aligned}
& \dot{x}=y, \\
& \dot{y}=\Psi(x, y),
\end{aligned}
$$

that starts at $(x, y)$ intersects the graph of the function $W$, and $\left(x_{s}, y_{s}\right)$ are the coordinates of that point of intersection. We make the convention that, if the initial condition $(x, y)$ is already on the graph of $W$, then $t_{s}=\infty$. A pair of trajectories of (4.4.2) and the graph of the function $V$ are shown in Figures 4.1 and 4.2, respectively. It should be mentioned that a differentiable Lyapunov function that leads to the construction of a continuous feedback that stabilises the double integrator (4.4.1) to the origin in finite time can be found in [10].

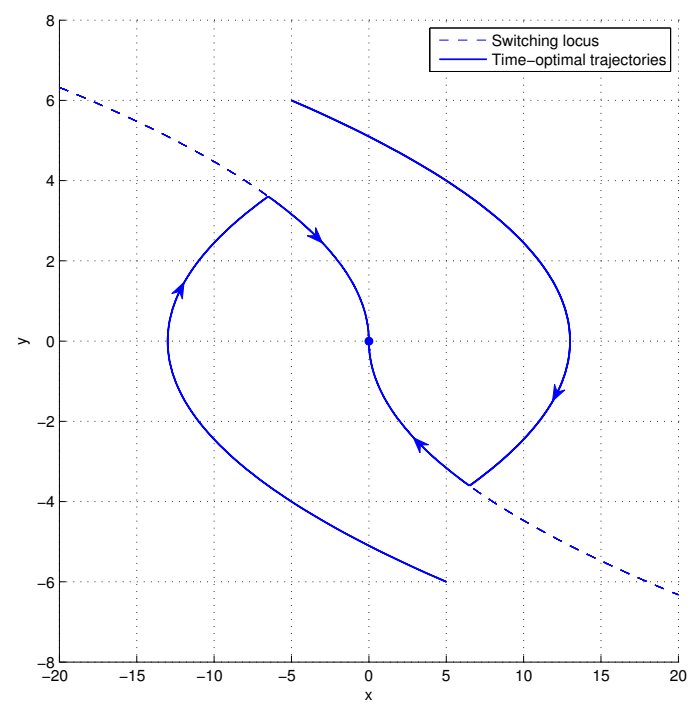

Figure 4.1. Two trajectories of (4.4.2) corresponding to initial conditions $(-5,6)$ and $(5,-6)$. 


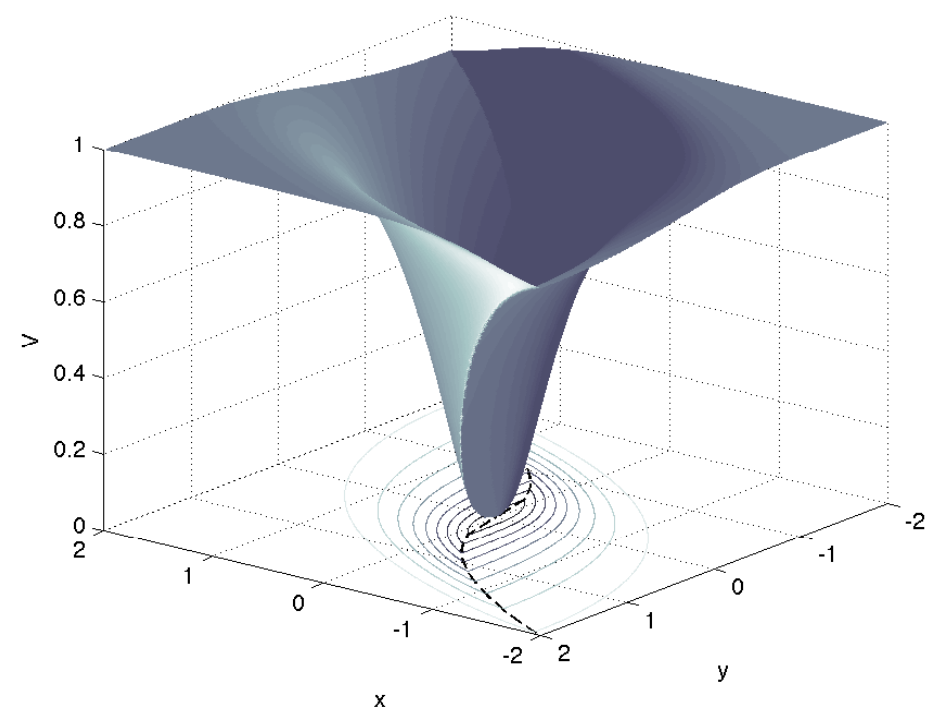

Figure 4.2. A numerical approximation of the Lyapunov function of Theorem 4.3 for system (4.4.2). 


\section{Chapter 5}

\section{Future work}

If we assume that the culmination of a general theory of stabilisation should be a taxonomy of control systems according to their stabilisability properties, then specifying the regularity of the feedbacks that can stabilise a given control system becomes a particularly important problem. It is not a problem of purely theoretical interest, however, and the examples in the Introduction (some of which we revisit below) demonstrate how it relates to concrete questions of stabilisability. The first section of the present chapter is devoted to examples and results from the literature that lead to the conclusion that regularity of feedback is an infinitesimal, rather than a local phenomenon. That is, it depends only on the properties of a control system at the equilibrium of interest and, therefore, should be studied accordingly.

The second section of the chapter is concerned with the gap that exists in the literature between the asymptotic notions of controllability and stabilisability and the analogous notions of finite temporal character. For example, geometric control theory focuses primarily on the notion of small-time local controllability, whereas the classical Lyapunov approach to stabilisation is mainly concerned with asymptotically stable closed-loop control systems. Establishing a connection between these two bodies of work that have been developing independently for decades will probably initiate an osmosis of ideas that could lead to further progress in understanding the structural properties of nonlinear control systems. The main point of Section 5.2 is to show how the first step in reconciling the finite and the asymptotic character of controllability and stabilisation can be precisely formulated in geometric terms.

The goal of the present chapter is a modest one, namely, to identify the right questions to ask in connection with the two problems mentioned above and described in more detail below. Their resolution is the matter of future work. Any claim of originality regarding the material of this chapter is bound to the presentation and not the results.

\subsection{Regularity of asymptotically stabilising feedback controls}

We saw in the Introduction and in Section 2.1.2 that Brockett's integrator, that is, the control system $\Sigma: \dot{\mathbf{x}}=u X(\mathbf{x})+v Y(\mathbf{x}), \mathbf{x}=(x, y, z) \in \mathbb{R}^{3}, X, Y: \mathbb{R}^{3} \rightarrow \mathbb{R}^{3}, u, v \in \mathbb{R}$, where

$$
X(\mathbf{x})=\left[\begin{array}{c}
1 \\
0 \\
-y
\end{array}\right] \quad \text { and } \quad Y(\mathbf{x})=\left[\begin{array}{c}
0 \\
1 \\
x
\end{array}\right]
$$


cannot be asymptotically stabilised at the origin by means of a continuous feedback control. However, it was observed in [29] that the feedback control given by

$$
\left[\begin{array}{l}
u \\
v
\end{array}\right]=\left[\begin{array}{cc}
x & -y \\
y & x
\end{array}\right]\left[\begin{array}{c}
-1+\frac{z^{2}}{\left(x^{2}+y^{2}\right)^{3 / 2}} \\
\frac{-z}{\left(x^{2}+y^{2}\right)^{1 / 2}}
\end{array}\right]
$$

and defined to be zero at zero, i.e., $(u(0,0,0), v(0,0,0))=(0,0)$, asymptotically stabilises $\Sigma$ on

$$
\mathbb{R}^{3} \backslash\left\{(x, y, z) \in \mathbb{R}^{3} \mid\left(x^{2}+y^{2}=0\right) \wedge(z \neq 0)\right\} .
$$

That is, there exists a feedback control which is continuous off the negative and positive $z$ semiaxes, and asymptotically stabilises Brockett's integrator at the origin $0 \in \mathbb{R}^{3}$. Similarly, it is shown in [29] that the feedback control

$$
u(x, y)=\left\{\begin{array}{lrl}
y^{1 / 3}+\frac{1}{3}\left(5 y+x-2 x^{3}-\frac{x^{4}}{y}\right), & y \neq 0 \\
0, & (x, y)=(0,0)
\end{array}\right.
$$

asymptotically stabilises on

$$
\mathbb{R}^{2} \backslash\left\{(x, y) \in \mathbb{R}^{2} \mid(x \neq 0) \wedge(y=0)\right\}
$$

the control system $\Sigma: \dot{\mathbf{x}}=X(\mathbf{x})+u Y(\mathbf{x}), \mathbf{x}=(x, y) \in \mathbb{R}^{2}, X, Y: \mathbb{R}^{2} \rightarrow \mathbb{R}^{2}, u \in \mathbb{R}$, where

$$
X(\mathbf{x})=\left[\begin{array}{c}
0 \\
y-x^{3}
\end{array}\right] \text { and } Y(\mathbf{x})=\left[\begin{array}{l}
1 \\
0
\end{array}\right] .
$$

Recall from Section 1.2 that the linearisation of (2.2.1) is not stabilisable and, hence, a stabilising feedback $u \in C^{1}\left(\mathbb{R}^{2}\right)$ for $(2.2 .1)$ does not exist. However, the feedback control (5.1.2) is real analytic off the $x$-axis. Both feedback controls (5.1.1) and (5.1.2) are obtained in [29] by first performing coordinate transformations that are known in the dynamical systems literature as "blow-ups" [32]. A question that naturally emerges from the analysis of the previous examples is the following [29].

Problem Let $f: M \times \Omega \rightarrow T M$ be a control system that is not continuously stabilisable at a point $p \in M$. That is, there does not exist $u \in C^{0}(M ; \Omega)$ such that $f(., u()$.$) is$ asymptotically stable at $p$. Is it possible to identify "singular submanifolds" of $\mathrm{M}$ off which a continuous stabilising feedback can be defined?

In the case of control-affine systems, a positive answer to the above question would be in agreement with the result in [70] where it is shown that asymptotically controllable (controlaffine) systems can be stabilised by means of feedback controls that are continuous outside closed sets of measure zero.

If a control system can be stabilised using continuous feedback, then, by virtue of Theorem 2.10, the obstructions to higher regularity, e.g., existence of smooth feedback, are concentrated at the equilibrium at which the control system is to be stabilised. For example, consider again the System (2.2.1). Since it was shown in Section 1.2 that (2.2.1) can be stabilised by a Hölder-continuous feedback, Theorem 2.10 implies the existence of an 
almost smooth control-Lyapunov function that can be used to construct an almost smooth stabilising feedback ${ }^{1}$ for $(2.2 .1){ }^{2}$ Similarly, since the control system $\Sigma: \dot{\mathbf{x}}=X(\mathbf{x})+u Y(\mathbf{x})$, $\mathbf{x}=(x, y) \in \mathbb{R}^{2}, X, Y: \mathbb{R}^{2} \rightarrow \mathbb{R}^{2}, u \in \mathbb{R}$, where

$$
X(\mathbf{x})=\left[\begin{array}{c}
0 \\
y^{2}-x^{4}
\end{array}\right] \text { and } Y(\mathbf{x})=\left[\begin{array}{l}
1 \\
0
\end{array}\right]
$$

can be stabilised by $C^{1}$ feedback [30], it can also be stabilised by a feedback which is almost smooth. However, it has been shown in [30] that no $C^{3}$ feedback can stabilise (B) at the origin. These observations lead us to the following question.

Problem Given a control system $f: M \times \Omega \rightarrow T M$ that is continuously stabilisable, is it possible to systematically specify the highest degree of regularity that a stabilising feedback for $f$ can have?

It is worth emphasising the obvious fact that the importance of the above problems stems from the interest to stabilise control systems for which we do not know a priori their stabilisability properties. For example, unless one is aware of the results in [30], it is not obvious why attempting to stabilise (B) using a parametrised polynomial feedback is a futile endeavour.

\subsection{The temporal aspect of stabilisation}

As mentioned in the introduction of this chapter, in the present section we formulate a question that, if answered, will provide a link between asymptotic and local controllability. We begin by recalling a few basic definitions.

Let $f: M \times \Omega \rightarrow T M$ be a real analytic control system and denote by $\mathscr{F}_{\Sigma}$ the collection $\left(f_{\omega}\right)_{\omega \in \Omega}$ of vector fields of the form $f_{\omega}: M \ni x \mapsto f(x, \omega) \in T M$. Let also $\mathscr{L}_{\Sigma}$ denote the Lie algebra generated by $\mathscr{F}_{\Sigma}$ and set

$$
\mathscr{L}_{\Sigma}(x)=\operatorname{span}_{\mathbb{R}}\left\{X(x) \mid X \in \mathscr{L}_{\Sigma}\right\}
$$

If $\mathscr{L}_{\Sigma}(p)=T_{p} M$, then the control system $f$ is said to satisfy the Lie algebra rank condition (LARC) at $p$. If a control system $f$ satisfies the Lie algebra rank condition at a point $p \in M$, then the reachable set $R_{f}(p,[0, T))$ has non-empty interior, for all $T \in \mathbb{R}_{>0}[82]$.

Suppose now that $f: M \times \Omega \rightarrow T M$ is a real analytic control system that satisfies the Lie algebra rank condition at $p \in M$ and is asymptotically controllable (AC) to $p$. Here we redefine asymptotic controllability to mean that, for any $q$ in a neighbourhood $U \subset M$ of $p$, there exists an admissible control $u$ such that $\lim _{t \rightarrow+\infty} x(t ; q, u(t))=p$. That is, we keep only (a local version of) the first part of the definition given on page 19, and the reason why we do so is because we believe it is important to isolate the defining properties of asymptotic

\footnotetext{
${ }^{1}$ Recall that an almost smooth function is a function which is $C^{\infty}$ in a punctured neighbourhood of $0 \in \mathbb{R}^{n}$. Since the set of control values $\Omega$ was assumed to have only the structure of a metric space, in order to extend the definition of almost smoothness to feedback controls we can either consider the special case $\Omega=\mathbb{R}^{m}$, or define a feedback $u: M \rightarrow \Omega$ to be $C^{r}$ if the vector field $f(., u()$.$) is C^{r}$.

${ }^{2}$ In other words, a corollary of Theorem 2.10 is that the existence of a continuous stabilising feedback implies the existence of an almost smooth stabilising feedback.
} 
controllability and understand separately their relevance to the problem we formulate in this section. Under the aforementioned assumptions on $f$, we examine the following two cases.

The control system $\boldsymbol{f}$ is STLC at $\boldsymbol{p}$. Then, given $T \in \mathbb{R}_{>0}, p$ lies in the interior of $\mathbb{R}_{-f}(p,[0, T))$, by Theorem 3.2 . Because $f$ is assumed to be asymptotically controllable to $p$, any point $q_{1}$ in a neighbourhood $U \subset M$ of $p$ can be steered in finite time to some point $q_{2} \in \mathbb{R}_{-f}(p,[0, T))$ and, by definition of $\mathbb{R}_{-f}(p,[0, T)), q_{2}$ can be steered to $p$ in finite time. Therefore, in this case, asymptotic controllability to $p$ and the Lie algebra rank condition at $p$, together, imply controllability to $p$ in finite time.

The control system $\boldsymbol{f}$ is not STLC at $\boldsymbol{p}$. Then $p$ has to be on the boundary of $\mathbb{R}_{-f}(p,[0, T))$, for all $T \in \mathbb{R}_{>0}$, and asymptotic controllability of $f$ implies that, for every $q$ in a neighbourhood $U \subset M$ of $p$, there exists an admissible control $u$ such that $\lim _{t \rightarrow+\infty} x(t ; q, u(t))=p$. In order to deduce controllability to $p$ in finite time it has to be shown that the control $u$ can be chosen in such a way that $x(t ; q, u(t)) \in \mathbb{R}_{-f}(p,[0, T))$, for some $t, T \in \mathbb{R}_{>0}$.

The previous considerations lead to the following question.

Problem Let $f: M \times \Omega \rightarrow T M$ be a real analytic control system that satisfies the Lie algebra rank condition at $p \in M$. Is it true that asymptotic controllability to $p$ implies local controllability from $p$ ?

It is reasonable to expect that an affirmative answer to this question will require a stronger notion of asymptotic controllability than the one we employ in this chapter, especially because of the "small-time" character of local controllability.

\section{References}

[1] E. H. Abed and J.-H. Fu. Local feedback stabilization and bifurcation control, I. Hopf bifurcation. Systems \& Control Letters, 7(1):11 - 17, 1986.

[2] E. H. Abed and J.-H. Fu. Local feedback stabilization and bifurcation control, II. stationary bifurcation. Systems \& Control Letters, 8(5):467 - 473, 1987.

[3] F. Ancona and A. Bressan. Patchy vector fields and asymptotic stabilization. ESAIM: Control, Optimization, and Calculus of Variations, 4:445-471, July 1999.

[4] F. Ancona and A. Bressan. Stabilization by patchy feedbacks and robustness properties. In M. S. de Queiroz, M. Malisoff, and P. Wolenski, editors, Optimal Control, Stabilization and Nonsmooth Analysis, volume 301 of Lecture Notes in Control and Information Sciences, pages 185-199. Springer Berlin / Heidelberg, 2004.

[5] Z. Artstein. Stabilization with relaxed controls. Nonlinear Analysis, Theory, Methods \& Applications, 7(11):1163-1173, 1983.

[6] A. Bacciotti. Local Stabilizability of Nonlinear Control Systems, volume 8 of Advances in Mathematics for Applied Sciences. World Scientific, 1992.

[7] A. Bacciotti and L. Rosier. Liapunov Functions and Stability in Control Theory. Communications and Control Engineering. Springer, 2005. 
[8] M. Bardi and I. Capuzzo-Dolcetta. Optimal Control and Viscosity Solutions of HamiltonJacobi-Bellman Equations. Systems \& Control. Birkhäuser, 2008.

[9] L. Berkovitz. Optimal Control Theory. Applied Mathematical Sciences. Springer-Verlag, 1974.

[10] S. Bhat and D. Bernstein. Continuous finite-time stabilization of the translational and rotational double integrators. Automatic Control, IEEE Transactions on, 43(5):678 -682, May 1998.

[11] S. Bhat and D. Bernstein. Finite-time stability of continuous autonomous systems. SIAM Journal on Control and Optimization, 38:751, 2000.

[12] N. Bhatia and G. Szegő. Stability Theory of Dynamical Systems. Classics in Mathematics. Springer, 1970.

[13] E. Bierstone and P. Milman. Semianalytic and subanalytic sets. Publications Mathématiques de l'IHÉS, 67(1):5-42, January 1988.

[14] V. Boltyanskii. Sufficient conditions for optimality and the justification of the dynamic programming principle. SIAM Journal of Control, 4:326-361, 1966.

[15] R. W. Brockett, R. S. Millman, and H. J. Sussmann. Differential Geometric Control Theory, volume 27 of Progress in Mathematics. Birkhauser, 1983.

[16] P. Brunovský. Every normal linear system has a regular time-optimal synthesis. Mathematica Slovaca, 28(1):81-100, 1978.

[17] F. Bullo, J. Cortés, A. D. Lewis, and S. Martínez. Vector-valued quadratic forms in control theory. In V. D. Blondel and A. Megretski, editors, Unsolved Problems in Mathematical Systems and Control Theory, pages 315-320. Princeton University Press, 2004.

[18] F. Bullo and A. D. Lewis. Geometric Control of Mechanical Systems, volume 49 of Texts in Applied Mathematics. Springer Verlag, New York-Heidelberg-Berlin, 2004.

[19] F. Camilli, L. Grüne, and F. Wirth. A generalization of Zubov's method to perturbed systems. SIAM Journal on Control and Optimization, 40:496-515, 2001.

[20] F. Camilli, L. Grüne, and F. Wirth. Control Lyapunov functions and Zubov's method. SIAM Journal on Control and Optimization, 47(1):301-326, 2008.

[21] F. Clarke. Lyapunov functions and feedback in nonlinear control. Optimal Control, Stabilization and Nonsmooth Analysis, pages 267-282, 2004.

[22] F. H. Clarke, Y. S. Ledyaev, E. D. Sontag, and A. I. Subbotin. Asymptotic controllability implies feedback stabilization. IEEE Transactions on Automatic Control, 42(10):1394-1407, October 1997.

[23] F. Colonius and W. Kliemann. The Dynamics of Control. Systems \& Control. Birkhäuser, 2000.

[24] J. Coron. Global asymptotic stabilization for controllable systems without drift. Mathematics of Control, Signals, and Systems (MCSS), 5(3):295-312, 1992.

[25] J. Coron and L. Rosier. A relation between continuous time-varying and discontinuous feedback stabilization. Journal of Mathematical Systems, Estimation, and Control, 4:67-84, 1994.

[26] J.-M. Coron. A necessary condition for feedback stabilization. Systems 8 Control Letters, 14(3):227-232, 1990.

[27] J.-M. Coron. On the stabilization of some nonlinear control systems: Results, tools, and applications. In F. H. Clarke and R. J. Stern, editors, Nonlinear Analysis, Differential Equations, and Control, volume 528 of Mathematical and Physical Sciences, pages 307-367. Kluwer, 1999. 
[28] J.-M. Coron and L. Praly. Adding an integrator for the stabilization problem. Systems \&6 Control Letters, 17(2):89 - 104, 1991.

[29] P. E. Crouch and I. S. Ighneiwa. Stabilization of non-linear control systems: the role of newton diagrams. International Journal of Control, 49(3):1055-1071, 1989.

[30] W. P. Dayawansa, C. F. Martin, and G. Knowles. Asymptotic stabilization of a class of twodimensional systems. SIAM Journal on Control and Optimization, 28(6):1321-1349, November 1990.

[31] J. Dieudonné. Éléments D'Analyse, volume II. Éditions Jacques Gabay, $3^{e}$ edition, 2005.

[32] F. Dumortier, J. Llibre, and J. Artès. Qualitative Theory of Planar Differential Systems. Universitext. Springer, 2006.

[33] L. C. Evans and M. R. James. The Hamilton-Jacobi-Bellman equation for time-optimal control. SIAM Journal on Control and Optimization, 27(6):1477-1489, 1989.

[34] R. Freeman and P. Kokotović. Robust Nonlinear Control Design: State-Space and Lyapunov Techniques. Modern Birkhäuser Classics. Birkhäuser, 2008.

[35] A. M. Gabrièlov. Projections of semianalytic sets. Functional Analysis and its Applications, 2(4):282-291, 1968.

[36] J. Gallier. Geometric Methods and Applications: For Computer Science and Engineering. Texts in Applied Mathematics Series. Springer, 2011.

[37] R. Gamkrelidze. Principles of Optimal Control Theory. Mathematical Concepts and Methods in Science and Engineering. Plenum Press, 1978.

[38] V. M. Gavrilyako, V. I. Korobov, and G. M. Sklyar. Designing a bounded control of dynamic systems in entire space with the aid of a controllability function. Automation and Remote Control, (47):1484-1490, 1987.

[39] R. Goebel, C. Prieur, and A. R. Teel. Smooth patchy control-Lyapunov functions. Automatica, (45):675-683, 2009.

[40] K. A. Grasse. On the relation between small-time local controllability and normal selfreachability. Mathematics of Control, Signals, and Systems, (5):41-66, 1992.

[41] K. A. Grasse and H. J. Sussmann. Global controllability by nice controls. In H. J. Sussmann, editor, Nonlinear Controllability and Optimal Control, volume 133 of Pure and Applied Mathematics, chapter 3, pages 33-79. Marcel Dekker, 1990.

[42] L. Grüne. Homogeneous state feedback stabilization of homogeneous systems. In Decision and Control, 2000. Proceedings of the 39th IEEE Conference on, volume 4, pages 3409 -3414 vol.4, 2000 .

[43] L. Grüne and F. Wurth. Computing control Lyapunov functions via a Zubov type algorithm. In Decision and Control, 2000. Proceedings of the 39th IEEE Conference on, volume 3, pages 2129-2134. IEEE, 2000.

[44] W. Hahn. Stability of motion. Grundlehren der Mathematischen Wissenschaften. Springer, 1967.

[45] R. M. Hardt. Stratification of real analytic mappings and images. Inventiones Mathematicae, (28):193-208, 1975.

[46] M. L. J. Hautus. Stabilization, controllability, and observability of linear autonomous systems. Indagationes Mathematicae, 73:448-455, 1970. 
[47] H. Hironaka. Introduction to real-analytic sets and real-analytic maps, 1973. Quaderni dei Gruppi di Ricerca Matematica del Consiglio Nazionale delle Ricerche.

[48] H. Hironaka. Subanalytic sets. In Number Theory, Algebraic Geometry and Commutative Algebra, in honor of Yasuo Akizuki, pages 453-493. Kinokuniya, Tokyo, 1973.

[49] R. M. Hirschorn and A. D. Lewis. Geometric local controllability: Second-order conditions. Proceedings of the 40th IEEE Conference on Decision and Control, pages 368-369, 2002.

[50] R. M. Hirschorn and A. D. Lewis. An example with interesting controllability and stabilization properties. Proceedings of the 45th IEEE Conference on Decision and Control, pages 3748-3753, 2006.

[51] B. Jakubczyk and W. Respondek. Geometry of Feedback and Optimal Control. Pure and Applied Mathematics. Marcel Dekker, 1998.

[52] V. Jurdjevic and J. P. Quinn. Controllability and stability. Journal of Differential Equations, 28(3):381-389, 1978.

[53] M. Kawski. Stabilization of nonlinear systems in the plane. Systems $\&$ Control Letters, 12:169$175,1989$.

[54] C. Kellett and A. Teel. Weak converse Lyapunov theorems and control-Lyapunov functions. SIAM Journal on Control and Optimization, 42(6):1934-1959, 2004.

[55] S. Krantz and H. Parks. A Primer of Real Analytic Functions. Birkhäuser Advanced Texts: Basler Lehrbücher. Birkhäuser, 2002.

[56] M. Krstić, I. Kanellakopoulos, and P. Kokotović. Nonlinear and adaptive control design. Adaptive and Learning Systems for Signal Processing, Communications, and Control. Wiley, 1995.

[57] J. Kurzweil. On the Inversion of Lyapunov's Second Theorem on the Stability of Motion, volume 24 of American Mathematical Society Translations. AMS, 1963.

[58] G. Lafferriere, G. Pappas, and S. Sastry. Hybrid systems with finite bisimulations. In P. Antsaklis, M. Lemmon, W. Kohn, A. Nerode, and S. Sastry, editors, Hybrid Systems V, volume 1567 of Lecture Notes in Computer Science, pages 636-636. Springer Berlin / Heidelberg, 1999.

[59] Y. Ledyaev and E. Sontag. A remark on robust stabilization of general asymptotically controllable systems. Johns Hopkins University, CiteSeer, 1997.

[60] E. B. Lee and L. Markus. Foundations of Optimal Control Theory. The SIAM Series in Applied Mathematics. John Wiley \& Sons, 1967.

[61] M. A. Liapunoff. Problème général de la stabilité du mouvement. Number 17 in Annals of Mathematics Studies. Princeton University Press, 1947.

[62] A. Loría and E. Panteley. Stability, told by its developers. In Advanced topics in control systems theory: lecture notes from FAP 2005, pages 199-258. Springer, 2006.

[63] J. L. Massera. On Liapounoff's conditions of stability. Annals of Mathematics, 50(3):705-721, 1949.

[64] J. L. Massera. Contributions to stability theory. Annals of Mathematics, 64:182-206, 1956.

[65] A. Michel, L. Hou, and D. Liu. Stability of Dynamical Systems: Continuous, Discontinuous, and Discrete Systems. Birkhauser, 2008.

[66] X. Mu and F. Tang. Uniform asymptotic controllability to a set implies semiconcave controlLyapunov function. In Proceedings of the Second International Conference on Innovative Computing, Informatio and Control, page 495. IEEE Computer Society, 2007. 
[67] H. Nijmeijer and A. J. van der Schaft. Nonlinear Dynamical Control Systems. Springer-Verlag, 1990.

[68] P. C. Parks. A. M. Lyapunov's stability theory-100 years on. IMA Journal of Mathematical Control and Information, 9(4):275-303, 1992.

[69] C. Prieur. Asymptotic controllability and robust asymptotic stabilizability. SIAM Journal on Control and Optimization, 43(5):1888-1912, 2005.

[70] L. Rifford. Semiconcave control-Lyapunov functions and stabilizing feedbacks. SIAM Journal on Control and Optimization, 41(3):659-681, 2002.

[71] L. Rifford. Stratified semiconcave control-Lyapunov functions and the stabilization problem. In Annales de l'Institut Henri Poincare (C) Non Linear Analysis, volume 22, pages 343-384. Elsevier, 2005.

[72] E. P. Ryan. On Brockett's condition for smooth stabilizability and its necessity in a context of nonsmooth feedback. SIAM Journal on Control And Optimization, 32(6):1597-1604, November 1994.

[73] C. Samson. Velocity and torque feedback control of a nonholonomic cart. In C. Canudas de Wit, editor, Advanced Robot Control, volume 162 of Lecture Notes in Control and Information Sciences, pages 125-151. Springer Berlin / Heidelberg, 1991.

[74] M. Shiota. Geometry of Subanalytic and Semialgebraic Sets, volume 150 of Progress in Mathematics. Birkhauser, 1997.

[75] E. Sontag. Feedback stabilization of nonlinear systems. Robust Control of Linear Systems and Nonlinear Control, 4:61-81, 1990.

[76] E. Sontag and H. J. Sussmann. Remarks on continuous feedback. In Decision and Control including the Symposium on Adaptive Processes, 1980 19th IEEE Conference on, volume 19, pages 916-921. IEEE, 1980.

[77] E. D. Sontag. A Lyapunov-like characterization of asymptotic controllability. SIAM Journal on Control and Optimization, 21(3):462-471, May 1983.

[78] E. D. Sontag. Mathematical Control Theory: Deterministic Finite Dimensional Systems, volume 6 of Texts in Applied Mathematics. Springer-Verlag, 1998.

[79] H. J. Sussmann. Single-input observability of continuous-time systems. Mathematical Systems Theory, 12(4):371-393, 1979.

[80] H. J. Sussmann. Subanalytic sets and feedback control. Journal of Differential Equations, 31(1):31-52, 1979.

[81] H. J. Sussmann. A general theorem on local controllability. SIAM Journal on Control and Optimization, 25(1):158-194, 1987.

[82] H. J. Sussmann and V. Jurdjevic. Controllability of nonlinear systems. Journal of Differential Equations, 12:95-116, 1972.

[83] M. Tamm. Subanalytic sets in the calculus of variation. Acta Math., 146(3-4):167-199, 1981.

[84] J. Tsinias. A general notion of global asymptotic controllability for time-varying systems and its Lyapunov characterization. International Journal of Control, 78(4):264-276, 2005.

[85] J. Warga. Optimal Control of Differential and Functional Equations. Academic Press, 1972.

[86] L. C. Young. Lectures on the Calculus of Variations and Optimal Control Theory. AMS Chelsea Publishing, 1980. 
[87] J. Zabczyk. Some comments on stabilizability. Applied Mathematics and Optimization, 19(1):19, 1989 .

[88] V. I. Zubov. Methods of A. M. Lyapunov and their Application. Groningen, P. Noordhoff, 1964. 


\section{Index}

$B^{n}(p ; \varepsilon)$ : a ball of radius $\varepsilon$ centred at $p \in \mathbb{R}^{n}$,

partition, 20 14

patch, 20

$R_{f}(p, t)$ : the set of points reachable from $p$ in time $t$ via trajectories of $f, 12$

$\mathbb{R}_{>0}$ : the interval $(0, \infty), 12$

$\mathbb{Z}_{>0}:$ the set of positive integers, 8

$\Gamma^{\omega} E$ : the vector space of real analytic sections of the bundle $E, 16$

almost smooth function, 10

Artstein's theorem, 10

Brockett's theorem, 6

control

admissible, 12

locally integrable, 12

piecewise constant, 12, 25

relaxed, 10, 19

control system, 12

asymptotically controllable, 19, 45

attractive, 17

controllable

locally, 12

small-time locally (STLC), 12

driftless, 17

locally continuously reachable in small time, 15

real analytic, 12

smooth, 12

control-Lyapunov function, 19

Coron's theorem, 9

diameter of partition, 20

equilibrium, 25

feedback, 20

asymptotically stabilising, 18

patchy, 21

piecewise analytic, 16, 24

relaxed, 10

s-stabilising, 20

time-varying, 18

Kawski's theorem, 13

Lie algebra rank condition (LARC), 45

negative orbit, 12

normal reachability, 25 